\title{
Processes of Science and Art Modeled as a Holoflux of Information Using Toroidal Geometry
}

\author{
Dirk K. F. Meijer \\ University of Groningen, Groningen, The Netherlands \\ Email: meij6076@planet.nl
}

How to cite this paper: Meijer, D. K. F. (2018). Processes of Science and Art Modeled as a Holoflux of Information Using Toroidal Geometry. Open Journal of Philosophy, 8, 365-400.

https://doi.org/10.4236/ojpp.2018.84026

Received: May 10, 2018

Accepted: August 7, 2018

Published: August 10, 2018

Copyright $\odot 2018$ by author and Scientific Research Publishing Inc. This work is licensed under the Creative Commons Attribution International License (CC BY 4.0).

http://creativecommons.org/licenses/by/4.0/

\section{(c) (7) Open Access}

\begin{abstract}
An attempt is made to model the structure of science and art discovery processes in the light of currently defined ideas on the societal flow of knowledge and conservation of information, using the versatile physical concept of toroidal geometry. This should be seen as a heuristic model that is open for further development and evolution. The scientific process, has been often described as a iterative and/or recurrent process. Current models explain the generation of new knowledge on the basis of a number of sequential steps (activities) operating in a circular mode. This model intrinsically assumes this process to be congruent for all individual scientific efforts. Yet, such a model is obviously inadequate to fully describe the whole integral process of scientific discovery as an ongoing interactive process, performed in a cumulative fashion. This implies that any new cycle starts from a different perspective or, optimistically seen, is initiated from a higher level, in a spiral mode, that takes into account the ongoing rise of scientific perspectives. Also, any model that attempts to picture the scientific process, should include potential interactions of concepts or hypotheses, in the sense that concurrently developed concepts may (mutually) influence each other and even may be mixed or superposed or, alternatively, may even result in concept extinction. Science and art progression, both seen as an individual effort and as a historically-based flow of events, is inherently a non-linear or even sometimes a chaotic process, where quite suddenly arising visions can cast a very different light on main-stream scientific thought and/or seem to remove existing barriers in more traditional "habits of the mind". In contrast to the rather gradual evolution of science, the history of art sometimes even shows complete rejection of preceding conceptualizations and styles. The dynamics of cognition and perception are fruitfully suggested by the rotational dynamics of a torus as a basis for its "self-reflexive" property. Also, the torus exhibits contraction/relaxation loops,
\end{abstract}


in which the torus turns inside out in a vibrating mode, implying strange loop trajectories. This suggests that the toroidal geometry embodies a cognitive twist, relating the "inside" to "outside" of knowledge as with a Möbius strip, a phenomenon that can be seen as the basis for self-consciousness. The torus geometry may also be applied to the art process on the basis of personal experience, intuitive vision, intention, imagination, and technical realization of the becoming piece of art. The finalization of the art concept can be conceived as a sort of knotting of the spiral information process: By literally connecting both ends of the toroidal information trajectory, the spiral is closed and a final product is created. Importantly, both scientists and artists may be inspired by intuition and serendipity, possibly through contact with an underlying knowledge field, as identified in modern physics. Unfortunately, science that often claims objectivity, sometimes seems dominated by a range of subjective human attitudes, not different from any other field in society. One factor is the deficient science-philosophical education of our students in the current curricula and loss of academic worldviews in university careers, in which "time is short" and necessary moments for reflection scarce.

\section{Keywords}

Toroidal Geometry, Art and Science, Scientific Discovery, Paradigm, Explanation/Inplanation, Science Inflation, Science Philosophy, Future of Science, Torus Knots and Art Products, Science Philosophy Education

\section{Science: Its Nature. An Introduction}

In the present paper, an attempt is made to model the structure of science and art discovery process, in the light of currently defined ideas on the societal flow of knowledge and conservation of information, using the versatile physical concept of toroidal geometry. This should be seen as a heuristic model that is open for further development and evolution. Current scientific methodologies do not provide a simple recipe for progress: Both science and art require intelligence, imagination, and creativity. In this sense, it is not sufficient to follow an automatic set of standards and procedures, rather science/art innovation seems to constitute an ongoing cycle of inquiry, constantly developing more useful, accurate and comprehensive models and also methods. For example, when Einstein developed the Special and General Theories of Relativity, he did not refute or discount Newton's Principia. Rather, Einstein's theories can be seen as expansions and refinements of Newton's theories, in which the latter, more classical natural science can be seen as nested in the current model of relative space-time and motion (see Wikipedia, Science method).

Usually the following elements (phases) are mentioned in current science methodology:

1) Define a well formulated question and/or develop a theory

2) Gather information and search for resources 
3) Form an explanatory hypothesis with some pertinent questions

4) Develop testable predictions of the particular hypothesis

5) Test the hypothesis by performing experiments and systematically collect data

6) Analyze the data and scrutinize them, if relevant by statistics

7) Interpret the data and draw conclusions that serve as a starting point for a new hypothesis

8) Publish the results in a transparent manner and/or communicate them otherwise

9) Retest the inferred concept (frequently done by other scientists)

The iterative cycle inherent in this step-by-step method goes from point 3 to point 9 and back again.

The scientific process, in the sense of current methodology, indeed, has been often described as an iterative and/or recurrent process. Current models, as the example depicted in Figure 1, attempts to explain the generation of new knowledge on the basis of a number of sequential steps (activities), operating in a circular mode. The model intrinsically assumes this process to be congruent for all individual scientific endeavors and discovery processes. Yet, such a model is obviously inadequate to fully describe the whole integral process of scientific discovery, as an ongoing interactive process of such individual efforts, being performed in a cumulative fashion.

Overall, the particular circular model, thus, is basically deficient also for a number of other reasons:

- In any cycle of conceptualizations, information is gained and, in general, knowledge increases (thus not a circular but rather a spiral format is at stake,

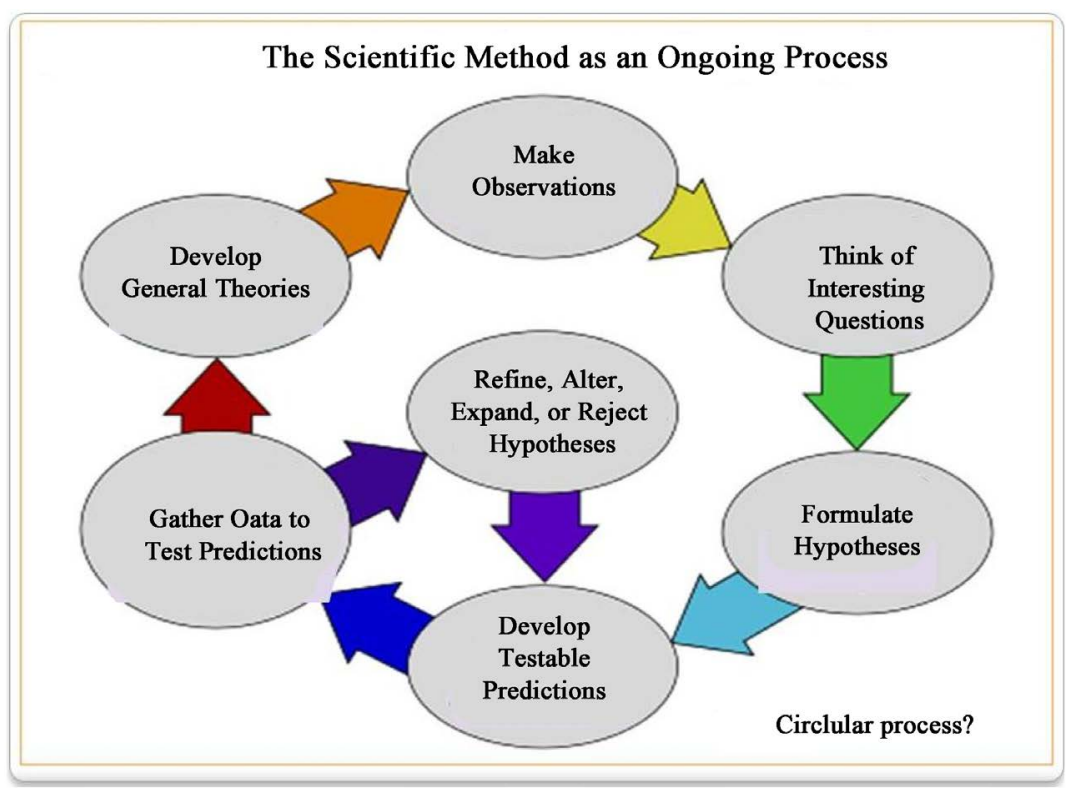

Figure 1. Example of a recurrent "Circular Model for Scientific Methodology", starting with making observations (oval, above middle), thinking questions, formulation of hypothesis, make testable predictions, gathering of data to test predictions, developing general theories that lead to new observations etc. 
see Figure 1). In the discovery process major negative results and solid rejections of hypotheses can occur, also through falsification of the particular theory, implying implicit setbacks to overcome. The ultimate knowledge may finally improve the scientist's overall picture and improve his/her scientific concept as well as their total scientific worldview. All this occurs against the background of the particular societal setting and it should be stressed that progress in science, historically seen, was virtually absent for long periods, both due to lack of technological advancement or through suppression by a dominant scientific establishment and/or religious institutes. Fortunately, contemporary science can be considered as a more continuous and cumulative process. This implies that any new cycle starts from a different perspective or, optimistically seen, is initiated from a "higher" knowledge level. It seems appropriate for this reason to disregard the recurrent cyclic model and replace it by a spiral modality that takes into account the ongoing rise of scientific knowledge and perspectives. Consequently:

- Any model that attempts to picture the scientific process should include potential interactions of concepts or hypotheses, in the sense that concurrently developed concepts may (mutually) influence each other and even may be mixed or superposed or alternatively may lead to concept extinction.

- The basic mechanism of information flow is determined by two major phenomena: an entropic force that produces a lack of information and disorder as well as a neg-entropic influence, through meaningful integration and compression of information. In these opposing elements central items of information processing, such as quality control of information and data correction are crucial. Of note, such a flow may also include information destruction/replacement, in order to remove excessive noise and information damping to minimize abundant side tracking.

- Any model for this process should take into account converging ("gravitational") and diverging ("anti-gravitational") influences on the generation of scientific data, for example due to the impact of external social influences (i.e. financing of research) and/or an essential availability of pertinent technology.

- Science progression has frequently been pictured as a classical Darwinian process (critically discussed by Wilkins, 1995) in which scientific theories undergo a sort of evolutionary process, including mutation, selection and "survival of the fittest", in a blindly operating setting without necessity of driving forces. Implicit in such an idea is the absence of teleology, with strong flavors of "self-aggregation" and emergence (the latter lacking predictive value, always being an explanation in retrospect). Yet, it is clear that in the science discovery process an essential driving force is apparent in the form of human curiosity, while goal-directed aspects are evident since the final aim of science is to fully understand nature and thereby ourselves (see later). Humans are not only observers of reality but also are active participants. They are not only part of biological evolution, but they directly give form to evolution and some even say that survival of the fittest is replaced by conservation of live-sustaining information. 
- In the creation of scientific thought, apart from rationality and sufficient overview of present scientific knowledge, subjective elements like serendipity, intuition and "feeling the future" can play a major role in scientific breakthroughs and technological innovations (Fishbein, 2005; Bernstein, 2005; Kastrup, 2017). Also the important role of directed creativity should be mentioned here (Plsek, 1996): Problem solving is an important activity, yet the primary indentifying and exposing of a hidden problem is obviously crucial in this respect.

- Progress in science requires a solid analysis of the process itself, implying careful observation from an appropriate intellectual "distance". This should include the views that are offered by scientific metaphysics. In relation to this it should be realized that science is a human faculty and that for a deeper penetration into the fabric of reality it is essential to understand how we observe and interpret with our individual memory as influenced by personal bias. It is urgent to obtain a better insight in the processes of observation, perception, and intuition in relation to the basics of human consciousness. Progress in conscious studies may therefore constitute a prerequisite for fundamental progress in science (Barus, 2001; Bohm \& Peat, 2008; Keppler, 2013; Filley, 2016; Meijer \& Geesink, 2017).

- The design of a scientific hypothesis requires both knowledge of the past as well as vivid anticipation of the future, related to the particular concept. This has much to do with imagination of how things could work out, in particular if cardinal changes would be made in the assumed prerequisites that underlie the present concept and that may block visions on potential innovation. Information, as a product of interaction thereby exhibits a clear dynamic and active character (see for the general aspect of information: See Meijer, 2012, 2013, 2014, 2015).

- In general terms, the building up of scientific knowledge can be viewed upon as a dynamic flow (or even better a flux) of information with an apparent cumulative character. This implies that an integral and accessible knowledge domain should be included (articles, reports, libraries, data banks, as also exemplified by the current dominant internet). In this respect the basic question has often been asked: where do new ideas actually come from? (Davies, 2006; Dodig-Crnkovic, 2007,2012 ). Are they spontaneously created by human intelligence, or do humans rather reveal already existing knowledge that is hidden in nature in the form of computational laws and should we regard "novel information" rather as an unfolding of premordial information (Barus, 2001; Bohm, 1987).

- Science and art progression, both seen as an individual effort and a historically-based flow of events, is inherently a non-linear or even sometimes a chaotic process (Perla \& Carifio, 2004), where even relatively small changes can lead to major scientific transformations. Instead of an ongoing "synthetic series of small steps, quite suddenly arising visions can cast a very different light on main stream scientific thought and/or seem to remove existing barriers in more traditional "habits of the mind". Such transitions or paradigm changes (see Kuhn, 
1970, in extensive discussions with Popper, 1969), have often been labeled as "science revolutions" (Newton, Darwin and Einstein being examples of unique leaders in this respect). Yet such revolutions seem to represent a rather scarce phenomenon (see further on; for general aspects on Scientific Change/Revolutions, see Nickles, 2014; Andersen \& Hepburn, 2016, and Scientific Revolutions, 2017 of. plato.stanford.edu.)

- During the course of history, one theory has succeeded another, and some have suggested further work while others have seemed content just to explain the phenomena. The reasons why one theory has replaced another are not always obvious or simple. The philosophy of science includes the question: What criteria are satisfied by a "good" theory. This question has a long history, and many scientists, as well as philosophers, have considered it. The objective is to be able to choose one theory as preferable to another without introducing cognitive bias (see Wikipedia, Models of Scientific Inquiry).

A proper theory shows typical features:

- Is elegant (formal elegance; no ad hoc modifications)

- Contains few arbitrary or adjustable elements (simplicity/parsimony)

- Agrees with and explains all existing observations (unificatory/explanatory power)

- Makes detailed predictions about future observations that can disprove or falsify the model if they are not borne out.

The desiderata of a "good or proper" theory have been debated for centuries, going back perhaps even earlier than Occam's razor, which often is taken as an attribute of a good theory. Occam's razor might fall under the heading of "elegance", the first item on the list, but was cautioned by Albert Einstein: "Everything should be made as simple as possible, but no simpler." It is arguable that parsimony and elegance "typically pull in different directions". The falsifiability item on the list is related to the criterion proposed by Popper, 1969 as demarcating a scientific theory from a non-scientific theory like astrology: both "explain" observations, but the scientific theory takes the risk of making predictions that decide whether it is right or wrong.

- The current science scene displays its own basic problems, by some called the "inflation in science". The particular, counter-productive, elements are generally very well realized by individual scientists, but often are not openly discussed in the scientific community. They include the lack of basic analyses of the scientific process itself, with the major influences of corrupted science policies, commercial interests, the power of traditional views and the lack of openness to novel approaches. All this seems related to the bare fact that science often claims a basic objectivity but rather seems dominated by subjective human attitudes, not different from any other fields in society.

Table 1 pictures some of these implicit personal aspects: Some of the various factors in Table 1 may, on their own, frustrate science progression, or in combination even can invalidate the very process of scientific exploration. 
Table 1. Current inflation in scientific endeavor.

o Each individual develops a worldview that is
more or less consistent
o The worldview is created by interaction of the
individual with the environment
o Worldviews are integrated in a mental
biography that is constantly adapted to
personal needs

o A worldview is an implicit part of self-esteem and thus of intellectual survival

o Personal worldviews tend to be intensely defended on the basis of their supposed meaning

o Scientific exploration always takes place within the context of a personal worldview

o Scientists have a worldview that is often claimed o Scientists are increasingly involved in money by them as objective and rational

o However scientists can never be fully objective and rational since their worldview is, per definition, personal and subjective

o Due to this field of tension, scientists look for moral support and professional consensus

o Consensus is always temporary and so are scientific theories and worldviews

o Scientists thus are striving for safety, a phenomenon that usually is found in mainstream science

o Scientist therefore tend to reject exceptions and refute anomalies, claiming to have a skeptic attitude o Scientist therefore tend to reject exceptions and refute anomalies, claiming to have a skeptic attitude

o Often skepticism takes a form of "moral space fright" and is poorly affected by self-criticism

o Scientists are often poorly educated in analyzing the processes they are part of themselves

o Some scientists identify alternative thinking as an attack on their personal integrity/worldview

o Some tend therefore to disqualify opponents or refrain from giving sufficient credits to them

o Alternative and innovative thinking, however, is an essential part of proper scientific endeavor o Some scientists loose independence, invalidating their societal task of critical professional assessor

o Scientific findings that are not in the interest of multinationals, sometimes become discredited in public

o Scientists in such an underlying position have no formal opportunity to submit their complaints

o Potential breakthroughs in science may (initially) be largely frustrated by a scientific establishment

Such detrimental effects can only be counteracted by continuous and critical monitoring of scientific quality as helped by open discussions on long-term aims, along the lines of ethics and professional attitudes (see also Eagleman, 2013). An amazing list of publications, aimed at this aspect, was published by the Institute of Venture Science, with rather critical comments on today's scientific enterprise. This comprehensive list, with no less than 150 critical but very professional articles of senior scientists, reveals an alarming situation in contemporary science and technology. Reading article headings such as: The Twilight of the Scientific Age, The Science Bubble, The Trouble with Science, Destroying Scientific Innovation, Rescuing US Biomedical Research from its Systemic Flaws, Why Most Published Research Findings Are False, Fund People Not Projects, Conform and Be Funded, Classical Peer Review: an Empty Gun, Mismeasurement in Science, Fake, Deceptive and Predatory science journals and conferences, and also the need of Repairing Research Integrity. See for further critical analyses of current science: Bauer, 2014; Huang, 2013; Bizzari, 2017; and Sare- 
witz, 2016.

Only inspecting the abovementioned titles already draws a quite dark picture of the state of art of current science, irrespective of discipline. In addition, one observes misuse of anonymous peer review in rejecting papers that are competitive or disagree with the opinion of the reviewer. Other current problems are the frequent decline in governmental research budgets, with the resulting take over by industrial interests, as well as publishers policies that frustrate open access publishing and many more calamities. All this seems to be accompanied by short term financial thinking and favoring shareholders interests, instead of investments in basic research. Especially counterproductive is the ongoing lack of real dialogue between the consensus/mainstream science and so called "anomalous" science (a distinction that science philosophical is quite nonsensical). Here we identify the special role of so called "skeptics", who are always equipped with the usual buzz words of statistics and "Occam razors", but are seldom critical toward their own criticism (see Skeptical investigations in ref. list). The general conclusion is of course that quite some scientists cannot have the pretention to be proper examples for the young. One crucial factor is the deficient science-philosophical education of our students in the current curricula and loss of the academic worldviews and fundamentals in university careers, in which time is often short and necessary moments of reflection scarce.

\section{The Science Process Modeled as a Toroidal Flux of Information}

In the following section an introduction will be given to toroidal geometry, since the ongoing processes of science and art, according to the author, can be envisioned as an interacting flow of concepts, ideas and hypotheses, that implicitly has a cumulative or progressive character (spiral representation), while the torus allows the modeling of such spiral processes of energy flow in a recurrent (self referential) modality. In the toroidal model the above-mentioned elements of scientific endeavor cannot only interact but also produce new, sometimes groundbreaking, concepts.

It should be stressed that application of the torus model is meant here, in the first place, as a metaphoric approach. Yet, it eventually could be developed into a physical description of the science, art and cognitive processes, since torus dynamics is generally regarded as an information integrator and also as a potential basic unit of quantized spacetime (see later).

The torus model is chosen since it is broadly used to describe the dynamics of physical processes at all scales of reality and in particular in quantizing space-time as a basic unit, Haramein et al., 2016; Bjerve, 2016; Meijer \& Geesink, 2017. The torus is supposed to represent a building block of space-time, in which (wave) information can be processed, stored and superposed (Figure 2). In this manner toroidal modeling can both be used as a metaphor in science philosophy and, in addition, for defining a physical space compartment that allows the 


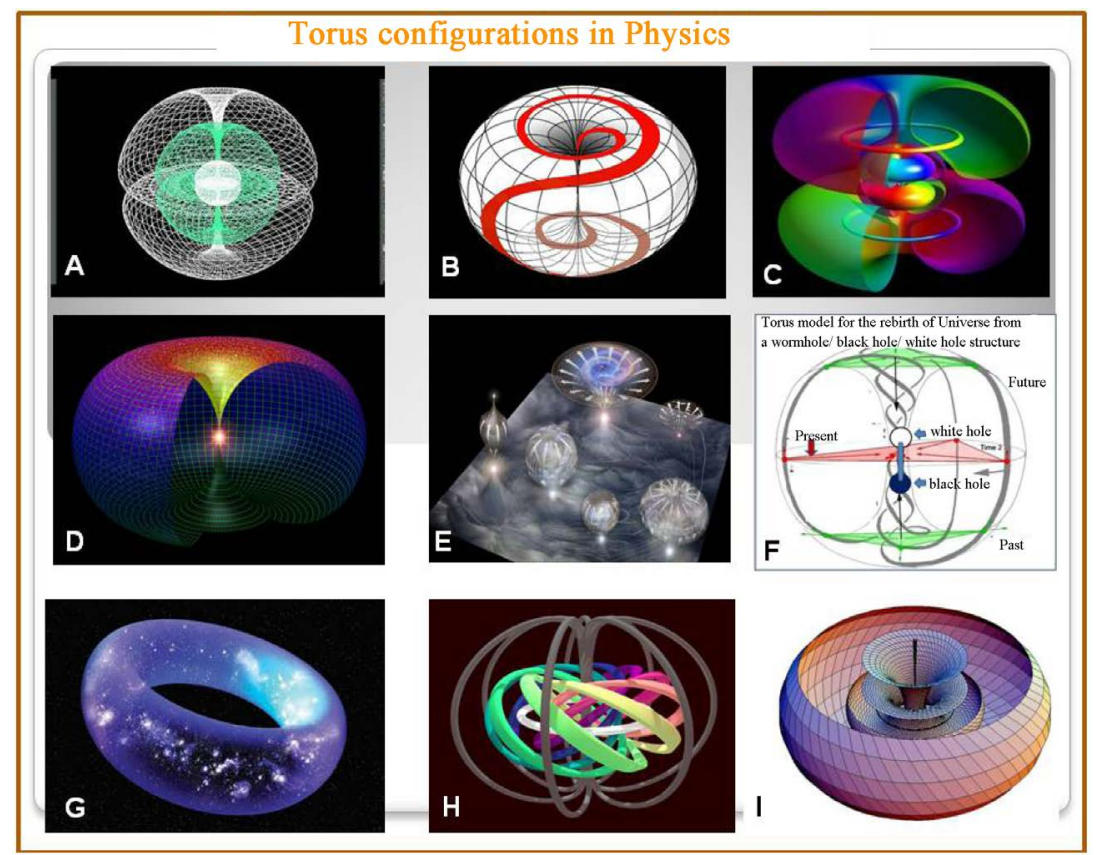

Figure 2. Various modalities of toroidal geometry: (A) Nested torus structure (B) Torus trajectory (red) (C) Atomic structure as double torus, (D) Filled space-time structure with singularity; (E) Torus network at the Planck scale: ultra-structure of quantum foam F: Torus model for rebirth of the Universe from a Wormhole/Black hole/White hole structure $(\mathrm{G})$ Donut model of the universe, $(\mathrm{H})$ Knot structure in torus as metaphor for attractor/standing wave, (I) cartoon of the twistor as a supposed space-time unit.

coupling of various modes of information (light, sound, complex information, information as meaning, meme plexes, ideas, concepts, physical laws, social settings in science and art, etc). In this sense the torus has been described as a "cognitive fusion reactor". The following features seem to be essential (see Meijer \& Geesink, 2016, 2017):

- Toroidal geometry enables to position the required spiral flow of information in a recurrent mode, mimicking the ongoing interactive science endeavor.

- From a geometric view the torus presents an extremely versatile rotating structure, allowing numerous information trajectories (Figure 3) with external (surface) and internal (core) components, meeting the complex nature and progressive aspects of science.

- The trajectories on the torus surface (Figure 3) implicitly introduce the aspect of time with aspects of past present and future (see Figure 6).

- The toroidal structure is equipped with the potential for wave interference, resonance, condensation and superposition, that can produce standing waves/ scalars, and thereby exhibits an integrative and innovative function, being a major feature of science.

- Due to its recursive and repeating aspect, both with regard to flow trajectories and various spinning modes, it offers the potential for (quantum) error correction and quality control as an essential aspect in scientific reporting and communication. 


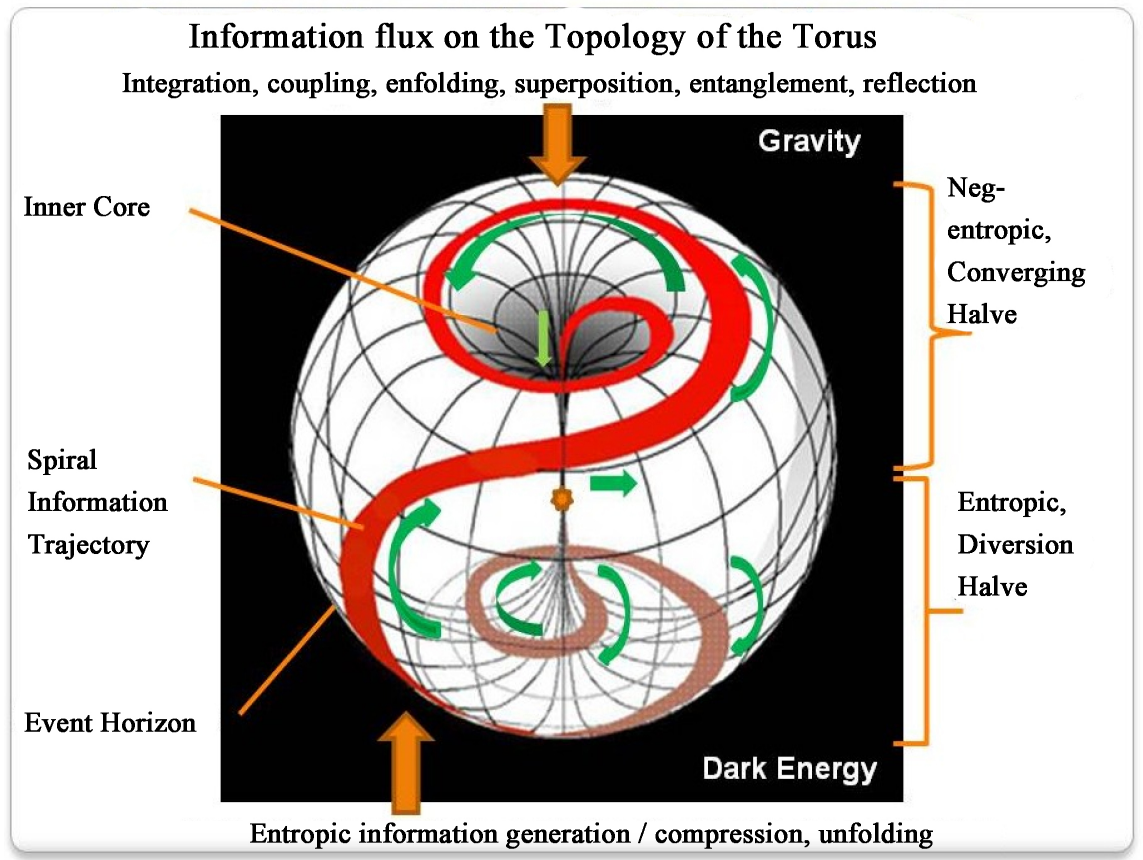

Figure 3. Processing of wave information using the topology of the torus: a spiral flow is depicted in the red trajectory that is initiated in the central core below and finally re-entering the core above. The lower halve of the torus displays an expanding (information entropic) activity that may be related to dark energy, whereas the upper halve exhibits the converging (gravitational) and neg-entropic aspect, that is symbolic for information compression. The outer topology of the torus can be envisioned as a holographic projection screen for projecting the information content of the torus. For interactive events within the inner core, see Figure 6 . In the central core processing of information includes aspects of integration, coupling, enfolding, superposition, entanglement and (photon/phonon) reflection of information. In each spiraling cycle (from bottom to top) knowledge is gained through concept interaction and quality control.

- The dynamic torus structure includes aspects of expansion and compression, that both are central in the generation and overview of information in the production of scientific data.

- The nested torus versions (Figure 4), enable the modeling of self-similar (fractal) scales that makes it possible to include sub-conscious aspects such as serendipity and intuition, being essential features of scientific discovery.

- The torus model can be expanded with an internal memory space that in principle allows information storage and retrieval that are prerequisites for building up a global knowledge field.

\subsection{The Torus as Information Integrator}

In order to explain what can potentially happen in the open core of the torus (Figure 2, Figure 3 and Figure 6), that is, if spiraling information quanta enter the central structure, many recent studies indicate that the toroidal structure allows the coupling of a whole spectrum of physical information carriers: photons, phonons, electrons as well as mixed forms of such wave/particles, such as 


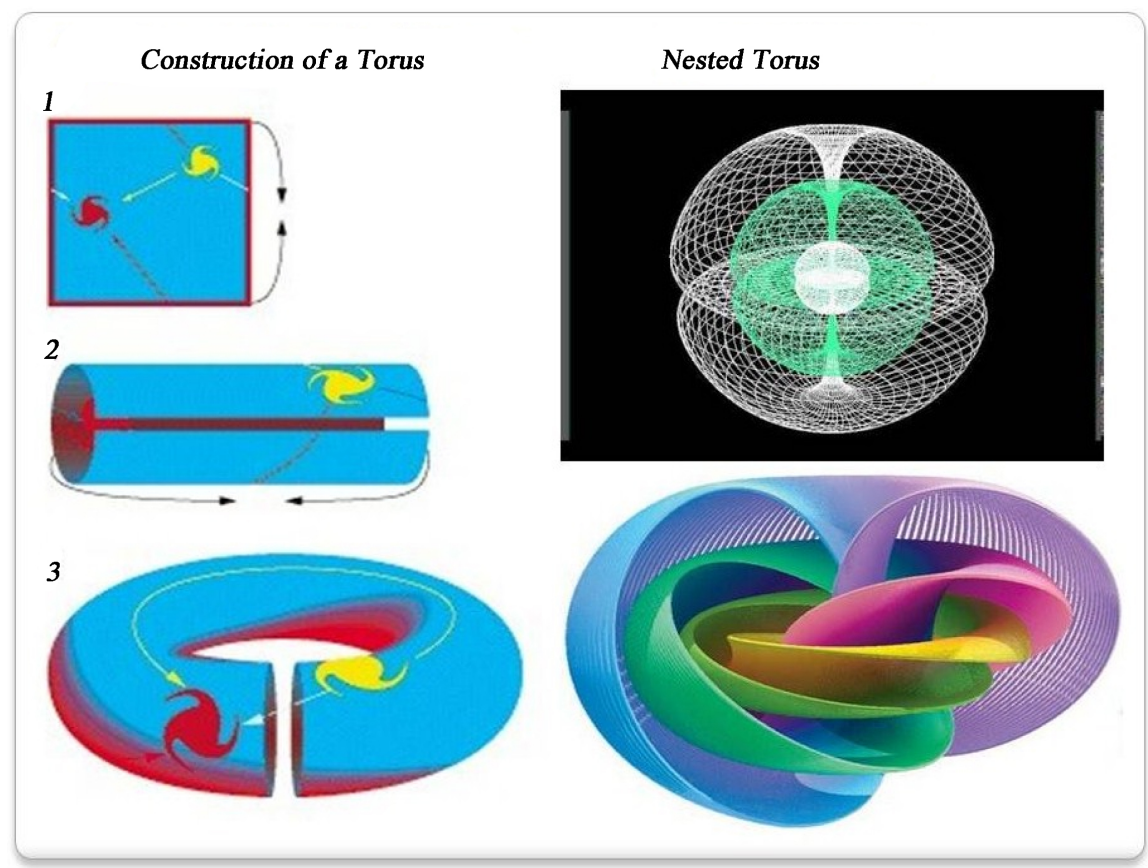

Figure 4. Construction of a torus (left) by connecting the two ends of a cylinder that was made from a simple plane, by which two wave functions can be repeatedly connected through a toroidal information flux. The nested torus structures (right) show multiple tori within the single torus. Right below: nested representation of fiber-filled torus (Hopf fibration), as also derived from torus geometry.

polaritons and polarons (Meijer \& Geesink, 2016). Due to the converging aspect of torus circuitry, these oscillations are converted to standing waves and/or scalar waves or may undergo condensation (see later). The scalar wave is the wave that remains when two opposite electromagnetic waves interfere, cancelling out the electric and magnetic field components. The resulting component in the electromagnetic wave, is the so called soliton, a non-linear longitudinal wave, vibrating in the same direction as it is travelling (Geesink \& Meijer, 2016a).

Another integrating activity, modeled by torus geometry, is that it can simulate the generation of a special collection of waves, that displays a similar vibration frequency, called coherent wave packages. A special form of this is known as Bose-Einstein conjugates. The latter have physically described only at very low temperatures, but more recently have been demonstrated to occur at much higher temperatures than assumed before. One example of this is the biological process of photosynthesis but various other coherent cellular processes are described now in the field of quantum biology (reviewed by Lambert et al., 2013). The particular coherent wave structures have potential influences on the organization of cellular organelles and cell water, as reviewed by Bishof \& Del Giudice, 2013 and Meijer \& Geesink, 2016.

Crucial in this respect is the intrinsic symmetry of the torus, showing a diverging (entropic) halve, in which waves on the surface expand and another part with converging properties, that stands for information integration and com- 
pression (neg-entropy). In cosmology, these two forces are defined as gravitational compression versus dark energy mediated expansion (see Figure 3). Scientific endeavor contributes to the ongoing (entropic) generation of huge amounts of information, whereas converging (integrative) activities occur in our brain, in addition to publication, review and internet spreading. Such activities continuously compress information, that by some is regarded as a physical metaphor, called gravitational (mutual attraction of mass). Both processes have been projected in torus models and assume that entangled wave information that enters the inner geometry of the torus core, can undergo potential non-destructive interferences and superposition.

It is important to note that such mechanisms can play a role at any fractal scale of reality, including the initiation and maintenance of life processes on our own planet. It was recently found that the spectrum of discrete EM frequencies that stabilize life conditions, may exhibit a quantum ordering effect on life cells on the basis of induction of geometric wave patterns (Geesink \& Meijer, 2016a, 2016a). The candidate radiation mode consists of coupled phonon/photon and electron wave energies, as well as quantum oscillations at far-infrared eigenfrequencies and were proposed to be communicated through a toroidal (constructive) interference into scalar wave information (Meijer \& Geesink, 2016).

\subsection{The Status of Toroidal Modeling in Physics}

The single torus can be considered basically as a surface of revolution, generated by revolving a circle in a three-dimensional space about an axis coplanar with the circle, in this manner in fact being a product of two circles. As treated in (Geesink \& Meijer, 2016a; Meijer \& Geesink, 2016), a torus consists of a central axis with a vortex at both ends and a surrounding coherent field. Energy flows in one vortex, through the central axis, out the other vortex, and then wraps around itself to return to the first incoming vortex (see Figure 3 and Figure 4). Topology is the science of form modulations.

The torus is usually seen as the fundamental form of balanced energy flow, found in sustainable systems at all scales (see later). Rotations projected as information flow in trajectories on the surface of the torus, can occur left and right handed as well as outside or through the central hole, indicating 3-D geometry. The 4-D modus of the torus is attained by an extra rotation: while a $3 \mathrm{D}$-torus can be envisioned as a cycling circle, a 4-D structure (Tozzi \& Peters, 2016), introduces a third cyclic element that rotates the torus inside out, as initiated by the internal negative curvature plane like an alternate explosion and implosion (see Figures 2-4) and ref. Judge, 2016a.

The torus geometry has been extensively used in physics to model the topology and presumable internal structure of elementary particles, such as the photon, which is seen as carrier of physical information with regard to wave/particle interactions, as well as in describing the structure of the electron. Wheeler and Feynman have earlier modeled electrons as spherical inward and outward electromagnetic waves. As mentioned above, besides electrons, also photons are 
considered to have toroidal geometries (Williamson \& Mark van der, 1997; Yepez, 2004). Various modalities of (nested) tori (Figure 2 and Figure 4) have been applied in geometric aspects of string theories such as the superstring model, called the type IIa theory, which postulates that the 6 extra space dimensions are compactified around a 6-dimensional torus (or tori).

These nested torus geometries include, so called, twistors, proposed by Penrose as a mathematical means of defining building blocks of space-time, exhibiting a nested and vibrational structure (Hopf fibration, see Figure 2 I and Figure 4). Twistors, as a special case of tori, posses aspects of holonomy, entanglement and enable an approach to quatum gravity, without need for the many extra dimensions as usually required in string theory. Twistor mathematics enable to transform information in our local 3-D spacetime into geometric/mathematical representation in a 4-D setting that may underlie our daily reality, also called a spin-liquid or amplituhedron (see later).

\subsection{Torus Dynamics as Metaphor for Scientific Discovery}

As mentioned in the introduction, the application of the toroidal geometry on the present work is primarily meant as a metaphoric approach in an attempt to model the complex endeavor of scientific discovery. This process can be viewed upon as an interactive stream of interacting scientific information, resulting in ideas, hypotheses, concepts and theories. Yet, in order to fundamentally analyze this process, a wide spectrum of creative human activities have to be positioned in their underlying connections, in a time progressive mode. Such a metaphoric approach was earlier proposed to characterize the properties of, so called, memes (infective/contagious pieces of information) in the human brain, by pointing to the strong resemblance with the various stages of viral infection (Meijer, 2007).

Indeed, the meme concept has also been applied to the process of scientific discovery, in which interactors (scientists) play a role by constantly exchanging information in the form of replicators. The latter are conceived as entities or units of information, tentatively called memes due to the (superficial) resemblance to gene transmission, possessing a positive selection bias (see Heylighen \& Chielens, 2006). These memes, are conceived as transmitters of cultural information, and are composed of scientific representations that form meme-plexes as a metaphor for scientific concepts/theories. Memes even have heritable aspects, since they are often related to a whole lineage of preceding theories, and the ongoing adaption is aimed at greater fitness and survival time in the competitive field. They can, in seldom cases, even rise to the dominance of paradigm change, but it should be realized that theories, including their successors, are per definition incomplete and vulnerable and often an absolute change or "revolution" is not at stake. If somehow associative or emotionally attractive, memes "infect" either the unconscious or conscious parts of the scientist brain, they are subsequently replicated and transmitted to other individuals in an unchanged form or undergo a "quantum-like superposition" to be spread out in the scientific community as a mutated modality. Bruza and Busemeijer, 2012, employ 
quantum theory and its features for such metaphoric description in socio-dynamic context.

Summarizing. There are distinct reasons to choose the higher dimensional and super-symmetric aspect of the, so called, double vortex torus, (Figure 2 and Figure 4) for outlining the scientific process, instead of a more simple circular process:

- The torus topology, with its internal channel-like aspect (see Figure 3 and Figure 4), with various types of information carrying vehicles, allows the coupling of very different modalities of wave information such as photons, phonons, electromagnetic energy flow, mixed forms of these, so called polaritons and polarons, as well as acoustic and cinematographic data, thereby mimicking the large diversity of scientific information (written reports/articles, reviews, books, video's, internet data etc.).

- The torus appears to be a favored geometric structure in physics and is widely applied for the description of elementary particles from the micro- to macro-scale of the universe (see later).

- The torus model integrates the present time as resulting from past and future wave projections (see Figure 6). The very centre of the torus is often described as a so called singularity in which the total information is compressed in unity and can function as a register of information on the brink between black hole and white hole structures, in which the present is indeed the result of memories of the past and projections of the future. In the total science process it reflects its current state of art, as a virtual dynamic state, resulting from a multitude of concept interactions and human experiences. The latter are ultimately instrumental in formulation of laws of nature, present and future technologies and metaphysical visions of the intelligent species that habit our universe (Meijer, 2015).

- The torus structure has self-referential properties and displays a recurrent flow of information that returns to its ever growing dynamical self. The latter aspect being a defined feature of self-consciousness, that in its turn is an essential property of scientific development (Meijer \& Geesink, 2017).

- The torus represents unity in diversity (a protected piece of space-time within a configuration of various force fields) that is also widely used in philosophical literature in a metaphysical context.

It was recently pointed out by Langan, 2017, that, intrinsically, in the ongoing process of science endeavor, a metaphysical language will be created that finally should enable a global coupling of mind and physical reality in a language with sufficient expressive power to picture a comprehensive description of reality or even a "theory of everything". Such a higher order language should be able to explain itself and thus the biological origin of mental activities of scientists in which minds it exists. This implies that the universe would share a linguistic structure and dynamics of the theory provide a metaphysical language that can even take a mathematical format and was called Cognitive-Theoretic Model of the Universe (CTMU). Thus, the CTMU is a theory which says that reality is in 
fact a self-modeling universal language. Langan proposed that in such a teleological causation, ordinary events are predicated on the generation of closed causal loops distributing over time and space. This loop-structure reflects the fact that time, and the spatial expansion of the cosmos as a function of time, flow in both directions: forward and backward as well as outward and inward, in a new kind of medium or "manifold". This concept is very much in line with the present toroidal model approach, as depicted in the present Figures 6-8 and may reflect the ultimate integration of scientific and artistic endeavor.

\section{Art and Music Modeled by Toroidal Geometry}

Art and science, historically seen, have always been strongly connected, in the sense that both human activities attempt to expose and understand the complex structures and deep layers of nature, albeit with very different methodologies (Filley, 2016). Also science often makes use of artistic representations while contemporary art increasingly integrates science-technological instrumentation (Banschoff, 1998; Hamlin \& Séquin, 2009). The history of art varied through time and from one culture to the other. Sculpture for example was considered a major art form in the Western world, whereas in ancient China it was first regarded as a product of manual labor out of the lower classes but later as holy way to describe the harmony in nature, the later being dominant in classical science in Greece and Arabian world with the emphasis on geometry and mathematics. The appreciation for science and art is therefore largely dependent on the particular part of the world and the time frame we take into account. Several authors have payed attention to similarities but also fundamental differences between Science and Art (see for some adequate papers: Malina, 1968; Scaruffi, 2007; Mathijs \& Mosselmans, 1993 and Maeda, 2004).

In Table 2, a tentative scheme is made to shortly list aspects of scientific (biased as beta-science) versus artistic processes, although such a list of commons and differences is colored by personal experience, time dependent and bears all the intrinsic dangers of generalization. Brady, 2000, sees perception as the connecting principle of Art and Science, and perception is not knowledge about it but rather an immediate knowing of or direct grasp of the perceived object. In this, sub-conscious recognition and intention as well as finding an adequate metaphor to characterize the object, play an important role. He stipulates Goethe's remark: "we see only what we know", of course not implying that we never see anything new. So it is important to realize that our individual representations are superposed on the background of own personal memory space in which we become aware of what we are thinking, this by seeing the understanding reflected back on us in the perception.

However, science more often tends to integrate earlier established knowledge into novel theories, considering that any theory is basically incomplete. It is realized that science is often a process of small steps in which most theories will be tested and then reformulated or adapted. Only generally accepted "laws of na- 
ture" have a relatively long survival time, since they mostly rest on solid and validated concepts and are often supported by a mathematical basis. Table 2 lists some fundamental difference in science and art, although it should be realized that this comparison reflects a rather small time window, while in the era of digitalization and dominant internet information spreading, the interaction between art and science can potentially rapidly change (see later).

For example, architecture and decorative art took many concepts from science and technology such as symmetry and biological order and this was certainly the case for mathematics and geometric representations, as a basis for advanced decorations and music (Smoyer, 2005). Even poetry can be presented in geometric forms as in, so called, concrete poetry. Famous mathematicians were often passionate musicians and sought to reformulate natural phenomena into a more

Table 2. Differences and similarities of science and art.

\begin{tabular}{|c|c|}
\hline Scientific process & Artistic Processes \\
\hline Science shows aspiration to inspire & Inspiration in art leads to material manifestation \\
\hline Tries to reveal deeper layers of nature & Provides portals to magical dimensions \\
\hline Based on satisfying fundamental curiosity & Tries to render the familiar unfamiliar \\
\hline Acquires knowledge by probing experience & Expresses personal experience and knowledge \\
\hline Claims objectivity that denies human bias & Acclaims subjectivity that denies social context \\
\hline Aims at universal consensus (laws) & Stresses individual experience and perception \\
\hline Strongly based on common rules & Highlights freedom from the known \\
\hline Concepts exposed to professional evaluation & Evaluation of products by colleagues is seldom \\
\hline Based on previous knowledge & Historical context not primarily leading \\
\hline Build on experimental research & Based on expression of feeling and scrutinizing ideas \\
\hline Aims at a universal view of the world & Is based on personal view of the world \\
\hline Highly organized communication networks & Communication between artists more incidental \\
\hline Requires extensive preparation/study & Is also initiated with limited preparation \\
\hline Is highly orientated towards high-tech & Technical aspects are often traditionally based \\
\hline Societal impact is often long term & Cultural impact can be rapidly demonstrated \\
\hline Is largely structured via specialization & Often shows more holistic approaches \\
\hline Reward is shown in citations and prizes & Rewards are shown in public awards and media \\
\hline Science strategy follows money & Commercial art is rejected, yet high prizes are paid \\
\hline Scientific quality is aimed at by peer review & Quality more shown by public appreciation \\
\hline Results widely spread by publication & Results shown by local expositions and internet \\
\hline Shows hierarchy and scientific leadership & Questions authority and breaks rules \\
\hline Breakthroughs can be based on serendipity & Unconscious impulses drive intuitive approaches \\
\hline Aims at a higher truth (theory of everything) & Likes diversity, unorthodoxy and many truths \\
\hline Conscious for ethical aspects of science & Ethics should be honored, but no censorship \\
\hline Multidisciplinary approaches are favored & Gaps between art disciplines are often huge \\
\hline
\end{tabular}


refined and coherent language, while illustrating their concepts with complex masterpieces of art, that even tried to catch the feeling of extra dimensions. The self-similar fractal pictures of Escher represent an astounding example. While artists empirically discovered the laws of colors, current brain investigators study the emotions they produce and try to unravel the very nature of such qualia. Consciousness studies address the deeper levels of meditation, while painting was often a spiritual exercise to create a focus for meditation and dreaming.

Also the rhythmic patterns of rotational and counter-rotating elements, as depicted in the present study, also in relation to chaotic systems versus deterministic causality, were popular subjects of art (Perla \& Carifio, 2004). Contemporary artists often maintain a strongly motivated rejection of the traditional formal logic of science but, interestingly, seem to prefer utilizing quantum formats of reality and information, such as uncertainty and entanglement.

Both in art and science some sort of creativity process plays a role. The creative process has also been described as a circular process exhibiting a stepwise process that might also fit the artistic modality: The artist identity, vision, intention(s) and insight, the engineering of the art object, the building or construction of the art object, the exposition and use of the artistic product, that in its turn contributes to the identity of the artist (Figure 5). Every original idea is imaginative, because only imagination can trigger creativity. This is why imagination is just as essential in science and technology as in the arts of our world. The difference between these two pairs of fields is that in science and technology imagination is largely disciplined rather than free. What motivates science disciplines is the objective truth requirement. Yet, Pauli stated: "it is more important to have beauty in one's equations than to have them fit experiment...",

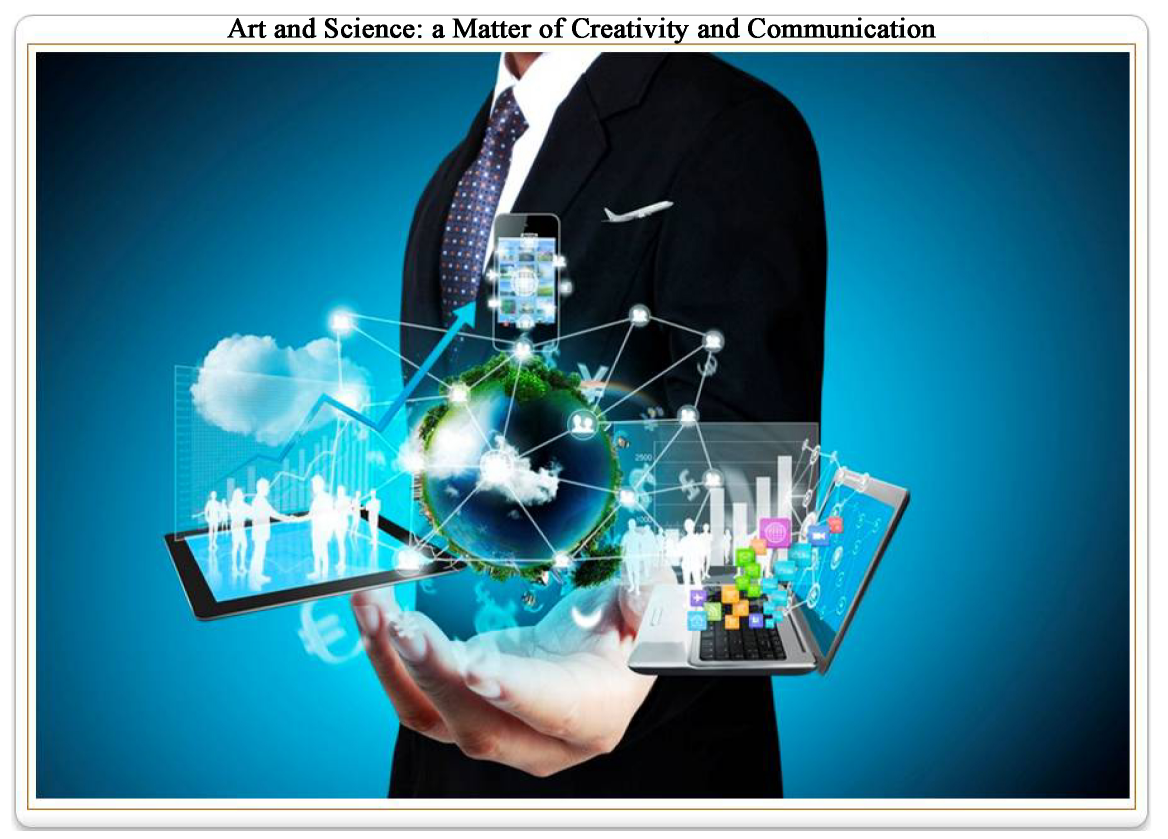

Figure 5. Science and Art depicted as an array of multi-level information, requiring creative thinking and proper communication of concepts and perspectives. 
indicating the appreciation of elegance of mathematical reasoning.

Revolutionary art at all times has served this function of preparing for the future. The faculty we use to grasp the nature of the "out there" is our imagination. Somewhere within the matrix of our brain we construct a separate reality created by a disembodied, thinking consciousness. This inner reality is unconnected to external space and exists outside the stream of linear time. This inner spectral vision amounts to a mental "opinion", unique to each individual of how the world works... When an entire civilization reaches a consensus about how the world works, the belief system seems to be elevated to the supreme status of a "paradigm," whose premises appear to be so obviously certain that no one has to prove them anymore. However paradigms intrinsically have a limited life time.

According to new physics, the observer and the observed are somehow connected, and the inner domain of subjective thought turns out to be intimately conjoined to the external sphere of objective facts. Through the complementarity of art and physics, these two fields intimately entwine to form a lattice upon which we all can climb a little higher in order to construct our view of reality. Understanding this connection should enhance our appreciation for the vitality of art and deepen our sense of awe before the ideas of modern physics. Thus art and physics, like wave and particle, can be seen as an integrated duality: They are simply two different but complementary facets of a single description of the world. Integrating art and physics will kindle a more synthesized awareness which begins in wonder and hopefully ends with wisdom.

Mathematics of the torus can be related to art in many ways. One can study art mathematically, looking for symmetries or other relations in the construction of a painting or sculpture. Conversely, mathematical algorithms can be used to help create art: fractal systems, for instance, can recreate realistic shapes of plants, mountains and clouds. Famously, perspective drawing has a mathematical basis, and is a good example of how different the human brain is from a digital computer. Perhaps one reason for this effectiveness of mathematics is that many laws of physics can be expressed in terms of minimizing free energy or minimizing action, that is also an important aspect of torus dynamics.

In the light of the abovementioned circular scheme of creative endeavor, the torus geometry may also be applied to the art process, and more particular in the artistic process on the basis of personal experience, intuitive vision, intention, imagination, and technical realization of the becoming piece of art. In the very process of design and making, a personal recurrent process of imaging, mental projection, intrinsic uncertainty, rejection of the initial concept, forced reconstruction, and possible feedback: they all picture the internal struggle of the artist. Yet, finally the pieces of the puzzle fall in place and not without compromises the choice is made to give birth to a final material or written representation of the initial impulse. The whole process can be envisioned as a flow of inner information fed by the dream or vision lying at the basis of the creative activity that always interacts with the outer world of instrumentation and potential reactions of the art spectators projected by the artist. 
In terms of torus modeling, the finalization of the art concept can be conceived as a sort of knotting of the spiral process: by literally connecting both ends of the toroidal information trajectory (Figure 6), the circle is closed and a final product is created. Earlier, Lacan, see ref. Gaudreau, 2016, modeled the human mind with three components: the real, the symbolic and the imaginary, by using a superposition of three tori. This requires, as earlier mentioned, the potential coupling of a large variety of mental and material information units, that in a recursive mode suddenly solidify in internally closed knot structure (comparable with a standing wave), in that each knotted structure embodies an created artifact (sculpture, painting, art object, poetry, book etc.).

Pieces of art are often unique and, in time, have a permanent character: they may be copied but are generally considered as a final product in the timeless form chosen by the artist. The variation in possible knots, that is on the basis of torus geometry, is huge: more than a million of possible knot variants have been mathematically derived. Non-trivial is the consideration that knotted structures can be unknotted or untied in a postulated 4th space dimension and multiple knots smoothly transformed into each other (Linov, 2014). In view of such metaphoric aspects: artifacts can be composed out of previous art objects and art concepts can be inherited or integrated and used as building blocks of novel artistic representations in general. In this respect it is interesting that knot theory have also been applied in the string-M theory and topology in physics (Kauffman, 1987),

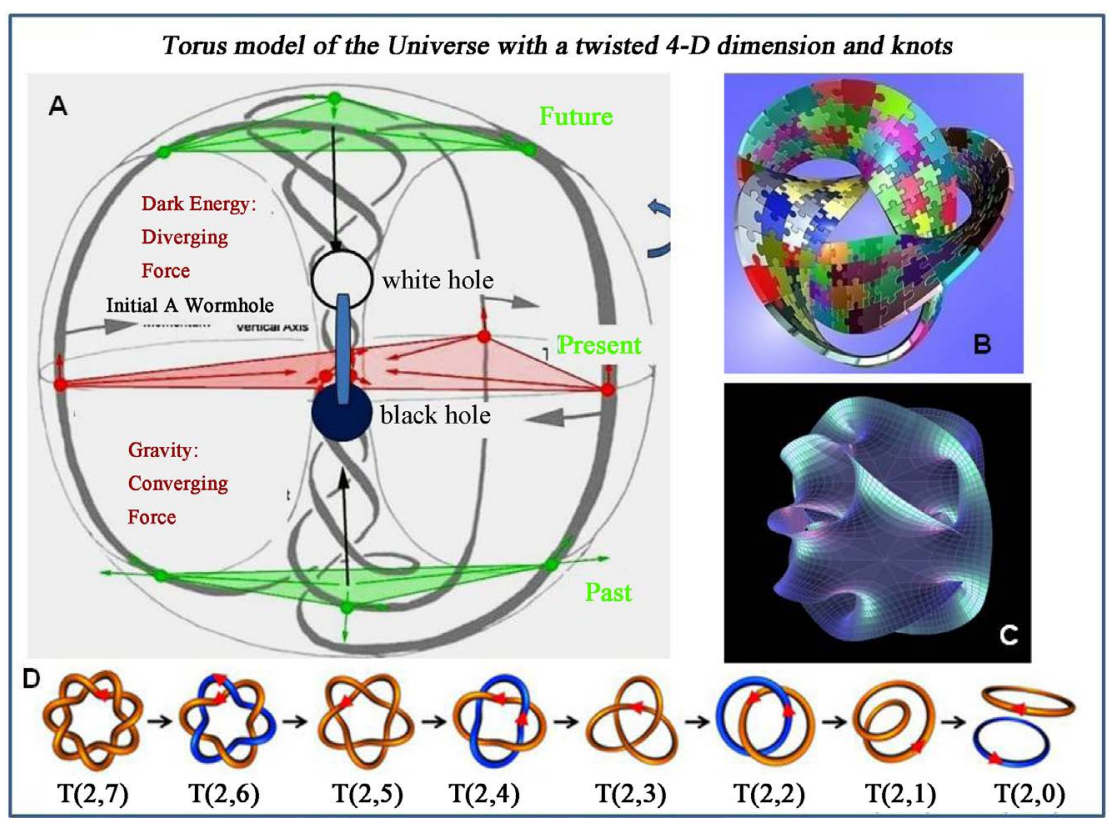

Figure 6. (A) Torus as a dynamic model for the recreation (rebirth) of our Universe from a wormhole structure, connecting an ultimate black hole (collecting and compressing all information) with a white hole (transmission and unfolding of information into a new version of the universe, see Meijer and Geesink, 2017; (B) Torus dynamics allow a twisted knotting into a 4-D space aspect of reality, C: Cartoon of Calabi-Yau manifold model of a multi-dimensional space (D) formation of various types of torus information knots, that may represent standing waves or attractors in the fabric of reality. 
and in particular in the quantizing of space-time. Works of art may be seen as heterogeneous building blocks of the total field of art.

With regard to the required inspiration of scientists and artists in their own way of analyzing/representing nature, it could be postulated that they both may derive essential information by intuitive mental contact (meditation, serendipity, synchronicity) with an information domain that is supposed to be underlying our three-dimensional local space-time geometry (Meijer, 2012, 2013, 2014). This dynamic electromagnetic field was proposed by David Bohm as an implicate order that through wave interference (so called pilot waves) guides matter in our common (explicate order) world. Bohm argued that we cannot directly observe this dimension but that we can experience it in listening to the waveworld of music with its wonderful mix of memorizing and anticipating harmonic wave patterns up to complete symphonies. Bohm \& Hiley, 1993, and Bohm \& Peat, 2008, did not provide a distinct physical or mathematical model for his implicate order, yet various followers did further substantiate this important idea (Holland, 1996; Sarfatti, 2015; Sutherland, 2006) by including a so called back-reaction in the information flux from our world to the ZPE energy field, through which this knowledge domain is permanently updated. It was soon realized that the all pervading quantum zero-point energy field could provide a fundamental basis for such a global memory space, since it can store physical wave information by polarization of its fluctuating quantum wave field (Figure 7).

Recently a hidden information domain beyond the Planck scale was theoretically revealed as a so called spin-liquid matrix. In relation to this it was earlier stipulated by Gough and Shacklett, 2014, that a knowledge domain beyond the Planck scale could provide characteristics of the well known implicate order of David Bohm. There is recent evidence that such a domain outside known spacetime could be constituted by a spin-liquid network, pictured as a geometric relations of information coined as the amplituhedron domain (Merali, 2017). Experimental support for a deeper information dimension beyond local space-time was found by Megidish et al., 2012, demonstrating that if one photon belonging to a pair of entangled photons is destroyed, entanglement between a newly created photon with similar features and the no longer existing photon can be detected, while these two photons never co-existed! This is strongly indicative for an information domain beyond our local 3D-space time in which information of the destroyed photon is somehow stored. Thus material particles in general should be seen as excitations of an underlying non-material matrix that behave as vortices or perhaps as tori in a 4-D setting. A deeper (geometric/mathematical) information domain was also implied in "Our Mathematical Universe" by Tegmark, 2014 (critically discussed by Butterfield, 2014). It was postulated by Keppler, 2016 and Meijer and Geesink, 2016, 2017 that our brain can convert ZPE wave information into attractor type of brain activity that may result in the conscious perception of archetypes/geometric forms that in their turn produce conceptions and innovative impulses in science and art (Meijer \& Geesink, 2017). 


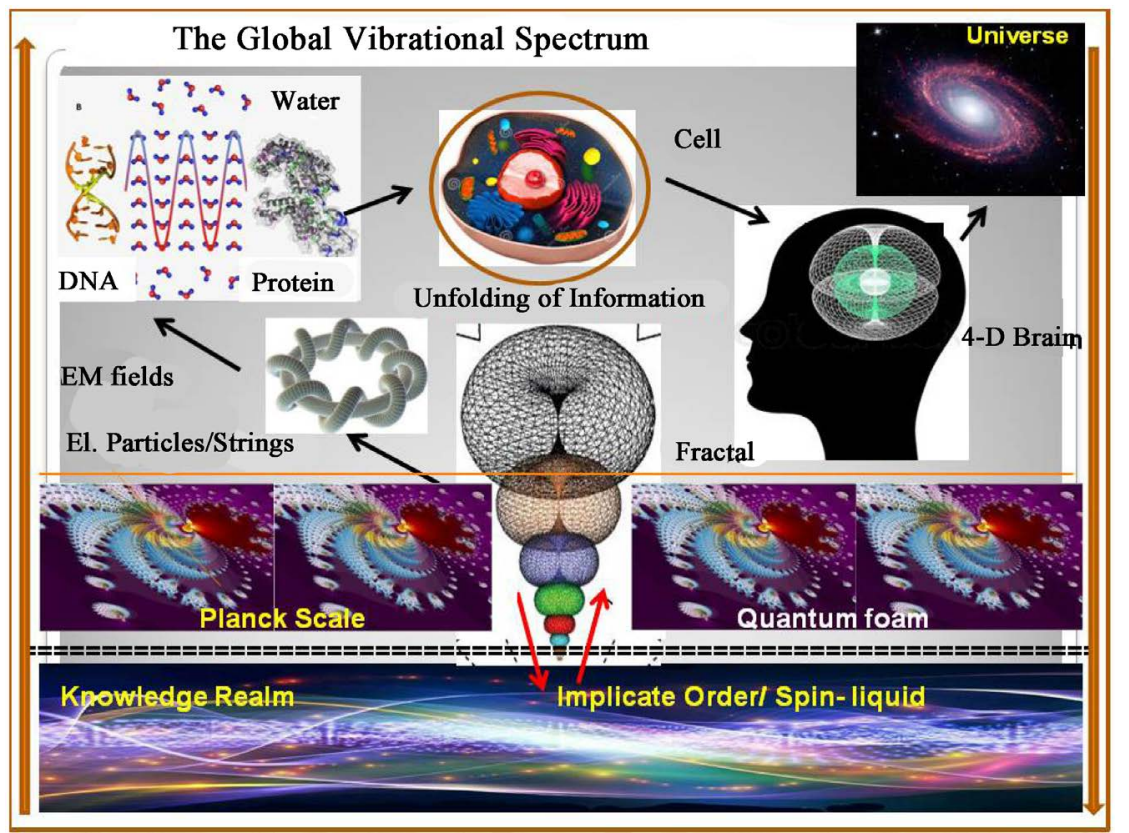

Figure 7. Morphogenesis of reality, seen as a fractal process of information unfolding, either (bottom up) from an implicate order frequency domain (below) up to the Planck scale, producing elementary particles, atoms, life molecules for the build-up of whole structure of the cells and organs such as the brain. All elements are proposed to be equipped with a toroidal memory workspace (consciousness) in a scale invariant manner. Universal information processing finally results in a black hole/white hole structure that (top-down) transmits information for the fabric of reality, thereby constituting a circular (rebound) mode of our universe (for bidirectional information flow, see large arrows at the two sides of the figure).

It is well known that various kinds of mental transcendence such as meditative states can inspire artists to see objects in very different perspectives. Such conscious states can also be induced by removal of an intrinsic neural "filter" in our brain that normally prevents such experiences, for example by certain programmed forced ventilation techniques or through use of psycho-active drugs (Kastrup, 2017).

In science and technology, major breakthroughs have be attained by intuition or serendipity in which, often in a relaxed state, sudden innovative solutions are revealed or new visions seem to be projected. This in, either a sort of inductive analysis or through a subjective emotional self-transcendence, sometimes felt as a form of personal clairvoyance. Wikipedia defines intuition as follows: "Intuition, a phenomenon of the mind, that describes the ability to acquire knowledge without inference or the use of reason". In the light of the evident importance of this aspect in scientific endeavor, it is surprising that so few systematic studies have been performed on methods to facilitate such productive mental states (Fishbein, 2005). One example is selective electromagnetic stimulation of certain parts of the brain (Kastrup, 2017). Another mechanism is the potential selection of our brain of discrete electromagnetic frequencies from the zero-point energy field (Lazslo, 2007, Meijer, 2012, 2013, 2014, as well as Keppler, 2013; Meijer \& 
Geesink, 2016, 2017), that were shown to induce coherent cellular processes in life systems in general (Geesink \& Meijer, 2016a, 2016b) as well as conscious perception through a field-receptive holographic workspace associated with the brain. Such a holonomic memory workspace bears toroidal features and may guide the functional binding of the various brain centers involved in conceptualization and awareness (Meijer \& Geesink, 2017).

\section{Torus as a Metaphor in the Creation of Poetry: Ex-Planation versus In-Planation}

The great Danish physicist Niels Bohr, in conversation with Heisenberg, remarked: "When it comes to atoms, language can be used only as poetry. What is not visible is, in effect, imaginary, and it is not possible to talk about such a thing without metaphors. A poem may be readily understood as a collection of verses-making of the poem a "multiverse" in its own right. Collections of poems can also be conceived as such. Poetry inspired by the complexities and highlighted by the imagination of physics was framed as quantum poetics.

A well known example, to illustrate the use of a metaphoric and archetypical figure in art and science, is the Ouroboros: a serpent that seems to swallow its own tail, bringing together the extremities of its body in a circular mode (see Figure 8). From ancient times it was seen as a symbol of eternal return and immortality. More recently, scientists such as Primack, 2006, projected the relative size of the defined structures in the universe on the snake body, going from the extremes of the smallest (so called, Planck) scale to the largest such as black holes and the entire universe. In this picture humans seem to take an intermediate (seemingly anthropomorphic) position, (see Figure 8, left). Yet, what do we actually really learn from this representation of cosmological scaling and the position that we, as human species, take in this respect? The inherent problem of the figure below is, that it takes the form of lengthy "explanation" at a time when there may be "recognition" of the need for "inplanation" (see Judge, 2016b). So called "explanations" in science often have the flavor of a satisfying reductionism and presuppose a certain consensus, thereby seemingly obtaining a rather definite character. In other words, they tend to escape the individual plane and, through this adverse effect, may not leave much space for future creativity. They seem to be resistant to the requirement of "future proof". Explanations, therefore, can effectively take people out of the plane of the reality in which they live, without ensuring that they are nourished by the deeper (potential holistic) perspectives offered. "In-planation", in contrast, could be understood as a necessary complementary process, enabling people to internalize and integrate knowledge (a sort of enfolding) in a more holistic way, information that can be later unfolded spontaneously and then centered in the here and now.

The process of "looking at" the cosmic Ouroboros, pictured above, can be seen as an explanatory trap in the case that it is only considered in a one-dimensional plane, even as depicted here as two dimensional figure. As mentioned above, 


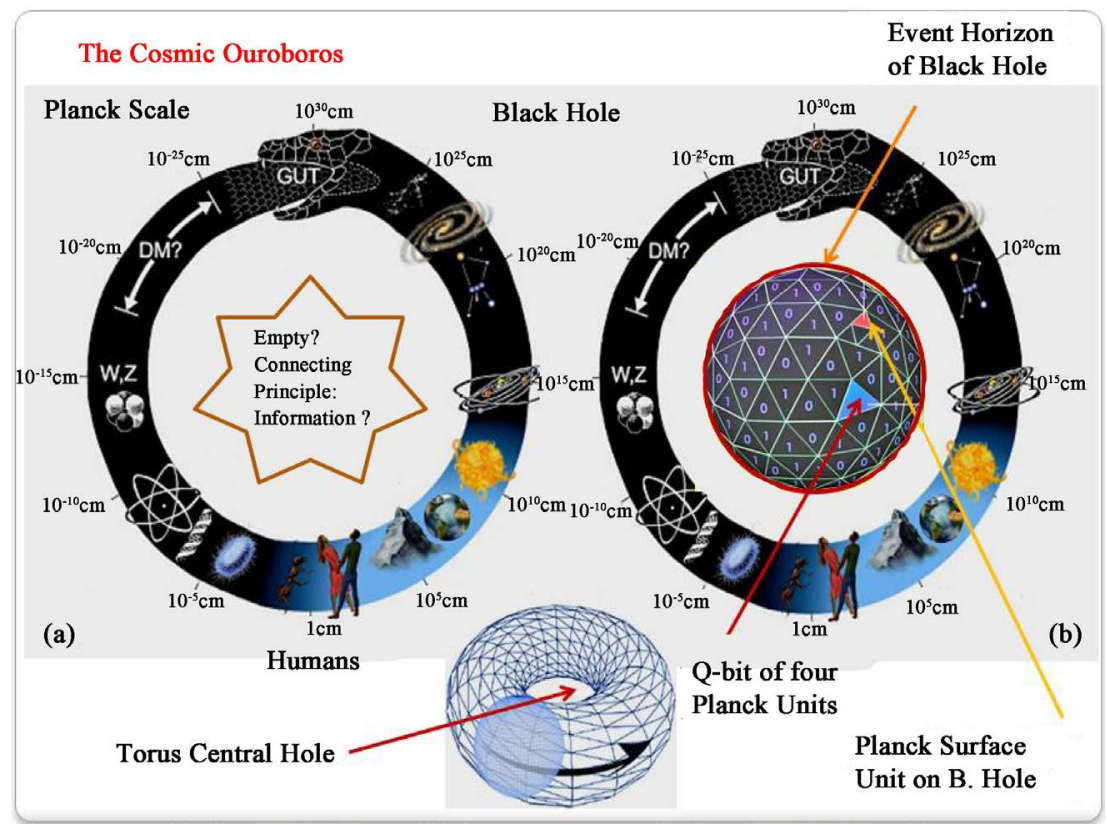

Figure 8. The Cosmic Ouroboros (a) The hollow ring structure formed by the snake can be interpreted as empty in the center, but alternatively can be seen as a flat torus exhibiting a central hole structure (middle below); (b) the alternative toroidal representation of the Ouroboros: the central hole is depicted here as a black hole spherical structure, on its surface bearing the basic units of information (in 0 and 1) that are projected on the black hole event horizon (red). Each Q-bit is composed of four Planck surface units. The cosmos at the right inset thereby contains a scale invariant information processing unit that is instrumental in global communication within and recreation of the universe.

the cyclic dynamics, along the body of the serpent, has recently been used as a mapping device for scaling the cosmos from micro- to macro-levels (Primack, 2006). However, no attention was drawn to any potentially circular dynamics around the serpent's body at each point along its length. The picture avoids to take into account the axis through the centre of the circle, along which the observer is located, as we know from the torus geometry treated above. Even more curiously the Ouroboros as pictured at the left of Figure 8, in a superficial way, takes the form of a zero, a container for nothing. Poetry (see below) expresses this elegantly:

And if the Wine you drink, the Lip you press, End in the Nothing all Things end in-Yes-Then fancy while Thou art, Thou art but what Thou shalt be-Nothing-Thou shalt not be less.

So what's missing? In the circular figure above to the right, we recognize a flat torus (a circling circle), with its known central channel-like axis or central hole. It is in this sense that the empty central portion of the Ouroboros constitutes a form for an "inplanatory process" (see later), effectively reflected as going "through" the plane of the Ouroboros as presented to the left (Judge, 2018b).

Now can we conceive the Ouroboros differently: not as an intoxicating bite or a suffocating swallowing of itself, showing a circular process of self death, but rather from a completely different perspective. This is realized by asking the 
question: what can the tail offer to the mouth so that the figure turns into a symbol of resurrection and ultimate physical rebirth as alchemists and philosophers like Jung told us long ago? Here we should employ deeper layers of the toroidal inner structure (representing the process of in-planation) that provides a superposition of wave information, recurrent coupling, and thereby information mirroring in a process of physical "self-reflection". In the framework of the cosmic Ouroboros in Figure 8, we now ask which element in nature can connect the various material shapes of the universe at the various scales?

A potential answer to this puzzle lies in the recognition of the central role of physical information, through which any of these structures can be described. The tail portion entails the smallest quantized (Planck) scale, supposedly composed of the smallest units of information: the Q-bit. The mouth portion of the serpent depicts the largest structure of black holes at the centers of all galaxies. Black holes not only gravitationally swallow information but, according to recent cosmological research, also radiates this information at their surface, called the event horizon. Here the smallest scale of the Universe meets the largest one known! In this model, the universe is conceived as a "living" structure that provides an event horizon-mediated back-radiation of conserved information (in Bits or Q-bits), arising from huge numbers of black holes that are likely distributed throughout the entire universe. This radiated, entangled, information is supposed to provide a holographic projection of all there is, including our own planet and its life forms. In the ultimate fate of the universe, a final single black hole, that contains the total of all generated information, will pass the accumulated information to a, so called, connected white hole. The supposed final black hole can in this manner function as a dedicated instrument for rebirth of our universe, in a cyclic operating mode (see Figure 6(A), treated in Meijer, 2015).

Now, realizing this, we may not longer see the cosmic Ouroborus as a "suicidal machine", but rather as an archetype that indeed symbols resurrection and eternity, beautifully expressed in the "Poetry of Life" by Jana F. White, 2013:

I don't fear melting into this earth.

Each morning

I wake into the air

I do not rise ... I do not move

I do not open my eyes

until my nose has sensed persuasion

my tongue has tasted sweetness

and my ears have heard the world

$\sim$

I was a child with dreams of becoming

Now I am older with dreams of being



Transformed 


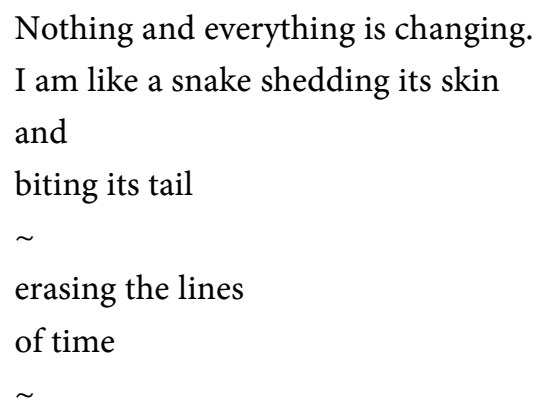

The dynamics of the process of in-planation represented by the torus as a mirror that calls for self-reflexive dynamics. In addition, the torus as a "Cognitive Fusion Reactor" exhibits contraction/relaxation loops, discussed earlier, in which the torus turns inside out in a vibrating mode, inviting strange loop trajectories. The "strangeness" of such a loop, see Hofstadter, 2007, further suggests that the toroidal Ouroboros is better understood as embodying a cognitive twist, relating "inside" to "outside" as with a Möbius strip (Sullivan, 2003). Of note, Stephen Hawking and colleagues (arxiv.org/abs/1205.3807) have shown that the universe may have the same toroidal geometry. Grossman, 2012 in New Scientist stated: "Hawking's 'Escher-verse' of the universe could be theory of everything".

\section{Attractors as a Product of Nonlinear Toroidal Information Flux}

Simple nonlinear dynamical systems can exhibit a completely unpredictable behavior, which might seem to be random, despite the fact that they are fundamentally deterministic. This seemingly unpredictable behavior has been called chaos. Here, the focus is not on finding precise solutions to the equations defining the dynamical system (which is often hopeless), but rather to answer questions like "does the long-term behavior of the system depend on its initial condition?" and also "will the system settle down to a steady state in the long term, and if so, and also what are the possible attractors?" If the torus dynamics is made up of a spiraling circle of information and complete one or more full revolutions, an attractor (see below) is produced that can be viewed upon as a sort of standing wave or scalar.

Similar attractors or standing waves can be hypothesized in the science process, since interference of very different theories can lead to unexpected outcomes, that seem to revolutionize the established worldview in the particular discipline. Yet, also seemingly small changes in existing theories, may produce large (non-linear) changes of view, as if chaotic systems (Perla \& Carifio, 2004). In addition, as treated earlier, serendipity (creative anticipation) or intuitive elements have to be taken into account. Such factors could only become approachable, if subjective (likely sub-conscious) information would be much better addressed. This, for instance, by future high resolution scanning of cognitive processes in the brain and/or through physical identification of mental connections to a supposed universal knowledge field, as postulated by Bohm \& Peat, 2008, Bohm \& Hiley, 1993, and Ervin Laszlo, 2007, not to mention many other 
authors. For this reason, major scientific breakthroughs would largely benefit from a better understanding of subjective (unconscious) perception in relation to intuition and serendipity.

Of note, the science transitions or paradigm changes, as intensively discussed by Kuhn and Popper (see Horgan, 2012), can only be approached by retrospect historical analyses. Yet, even if such phenomena could be inferred from the landscapes of science and art, so called "paradigm changes" are open to question, as was discussed, for example, in the case of Darwinian revolution (Ruse, 2009). Therefore the present author prefers the term science/art attractors, over expressions such as science paradigms, revolutions, turnovers and transitions.

\section{The Torus in Music Theory}

Music deals with the exploration of tension and resolution, and at the same time, anticipation. Music implies the control of complex sensations of sound into emotion and feeling. In this sense, music could be said to be more complete than science, for it seeks a harmony between the four basic human functions: thought balanced by feeling and intuition rising from sensation.

Toroidal geometry has been used in different ways by various music theorists (see Merrick, 2010) to describe the relationships between major and minor triads, called the Tonnetz torus (see insets Figure 9A and Figure 9B). Triangular lattices placed on the torus surface present identical musical constructs such as chords, regardless of the beginning pitch and show hexagonal geometry. The implicit principal transformations are expressed as minimal motions in the Tonnetz pattern, which can be seen as the wrapping of the planar graph onto a torus (Figure 9B). Sound induced geometric patterns can be induced by wave interference (Figure 9D) in, so called, Chladni experiments (Figure 9C and Figure 9E, see Meijer \& Geesink, 2016). Interestingly, so called, causal dynamic triangulation, have also been used for describing the holographic geometry of the universe and also a quantized Planck space (Loll, 2011).

Toroidal information integration and processing, interestingly, is also applied in tonal theory (Purwins et al., 2007) and recently in music studies of Van De Bogart and Forshaw, 2015. The latter authors showed that quantum algorithms can be coded through toroidian information compression, using frequency resonance, by which information can be encoded in electronic sound that in turn can be decoded to the original information. The particular information processing procedure resembles a self-generating imagination that exhibits probabilistic fractal features for storing and retrieving information, thereby attaining a sort of neuroplastic quality. Toroidal flow may, in this respect, be conceived as a modality of rotational information flux, that returns to itself, a characteristic that may be the very basic mechanism for creation of awareness and (self)-consciousness (Meijer \& Geesink, 2017).

It is generally recognized that from ancient times, music has played a crucial role in the making of modern science. This item was adequately covered in the 
The Harmonic Background in the Fabric of Reality : Wave Interference

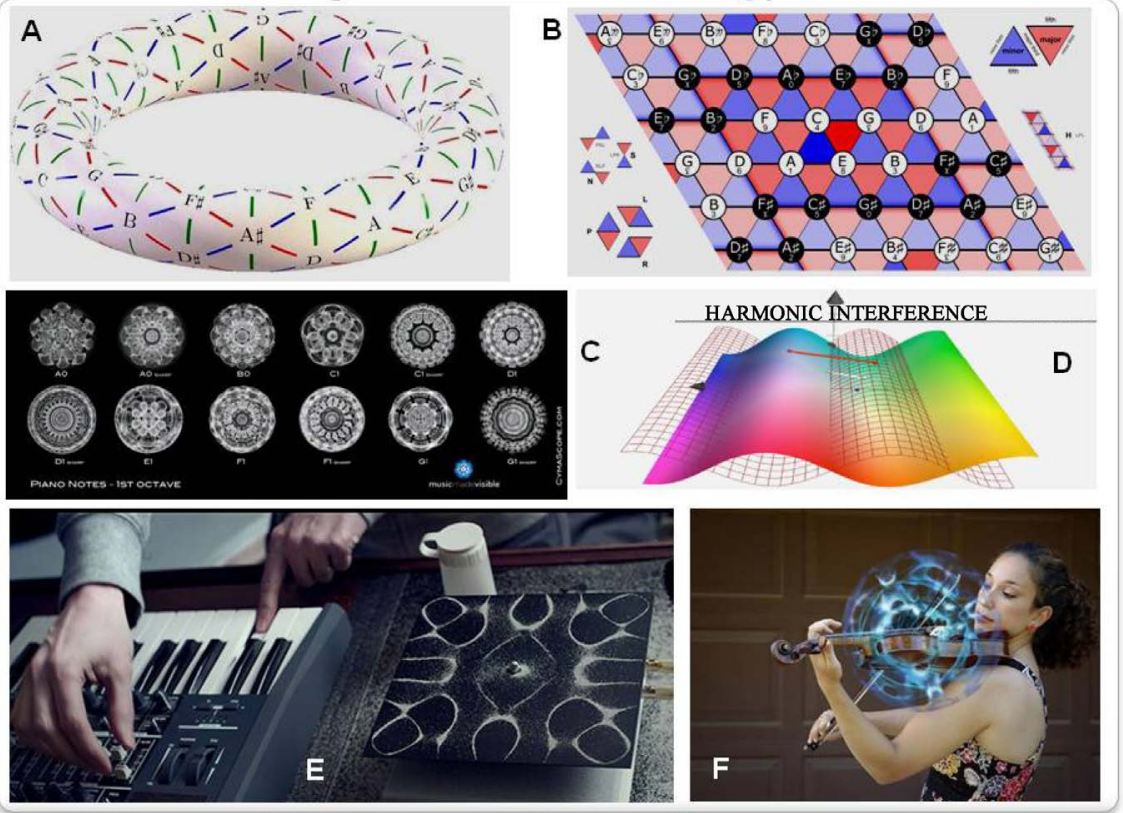

Figure 9. (A) Represents the so-called Tonnetz torus on which various pitches can be projected together with their interconnection that as spiral trajectories on the torus provide the particular melody; (B) Pitches in the tonnetz are connected by lines if they are separated by minor or major third or perfect fifth, the tonnetz having 12 nodes (pitches) and 124 triangles; (C) Complex geometric patterns that are produced on a sand-covered vibratory surface, exposed to musical vibrations (see (E) for a typical experiment); (F) Such geometric patterns may also be induced on a music instrument by pressure differences in the air that in this manner can reach our ears to evoke corresponding vibrations in our eardrums.

book of Peter Pesic, 2015, who stated that in the natural science of ancient Greece music formed the meeting place between numbers and perception. For the next two millennia, Pesic writes in Music and the Making of Modern Science, "liberal education" connected music with arithmetic, geometry, and astronomy within a fourfold study: the quadrivium. Peter Pesic argues clearly that music had a formative effect on the development of modern science, since music represents a conceptual force in its own right. Pesic explored a series of episodes in which music influenced science, moments in which prior developments in music arguably affected subsequent aspects of natural science. He describes encounters between harmony and fifteenth-century cosmological controversies, between musical initiatives and irrational numbers, between vibrating bodies and the emergent electromagnetism. He offered lively accounts of how Newton applied the musical scale to define the colors in the spectrum; how Euler and others applied musical ideas to develop the wave theory of light; and how a harmonium prepared Max Planck to find a quantum theory that reengaged the mathematics of vibration.

Taken together, these cases document the peculiar power of music, its autonomous force as a stream of experience, capable of stimulating insights different 
from those mediated by the verbal and the visual. Merrick, 2008, in his masterpiece "Interference Theory", used a toroidal model to describe music as a balance between resonance and damping. He stipulated, though, that harmonic science in history was long suppressed, especially by Western religion and later by mainstream science until quantum mechanics provided a complete change of mind. The latter is most clearly reflected on the current ideas of the Nobel laureate Frank Wilczek, 1989, 2016, in the article "Why physics needs art in picturing the universe" as well as in his books on "Longing for Harmonies", and " $A$ Beautiful Question, Finding Nature's Deep Design", respectively. Pitkänen, 2013, like David Bohm, suggested music as a basis to study consciousness. The present author, together with Hans Geesink, revealed strong evidence for the presence of a generalized music (GM) scale with a semi-harmonic character of discrete EMF frequency bands that seem to underlie a spectrum of animated and non-animated biophysical phenomena, including the quantum standard model of particles (Geesink \& Meijer, 2017a, 2017b; Geesink \& Meijer, 2018), very much in line with the notions of Niemetz, 2004. Interestingly, the torus model was extensively used in the latter study as have also been done before by Merrick, 2008; Bentov 1979; Young, 2006, and was also expressed in the articles of Barr, 2006 and Haramein et al., 2016.

\section{The Future of Science and Art in a Toroidal Cyberspace}

Rosen (2005) compared real space, mental space, and virtual electronic space that connect them. His claim was that the three topological spaces (see Figure 10E)

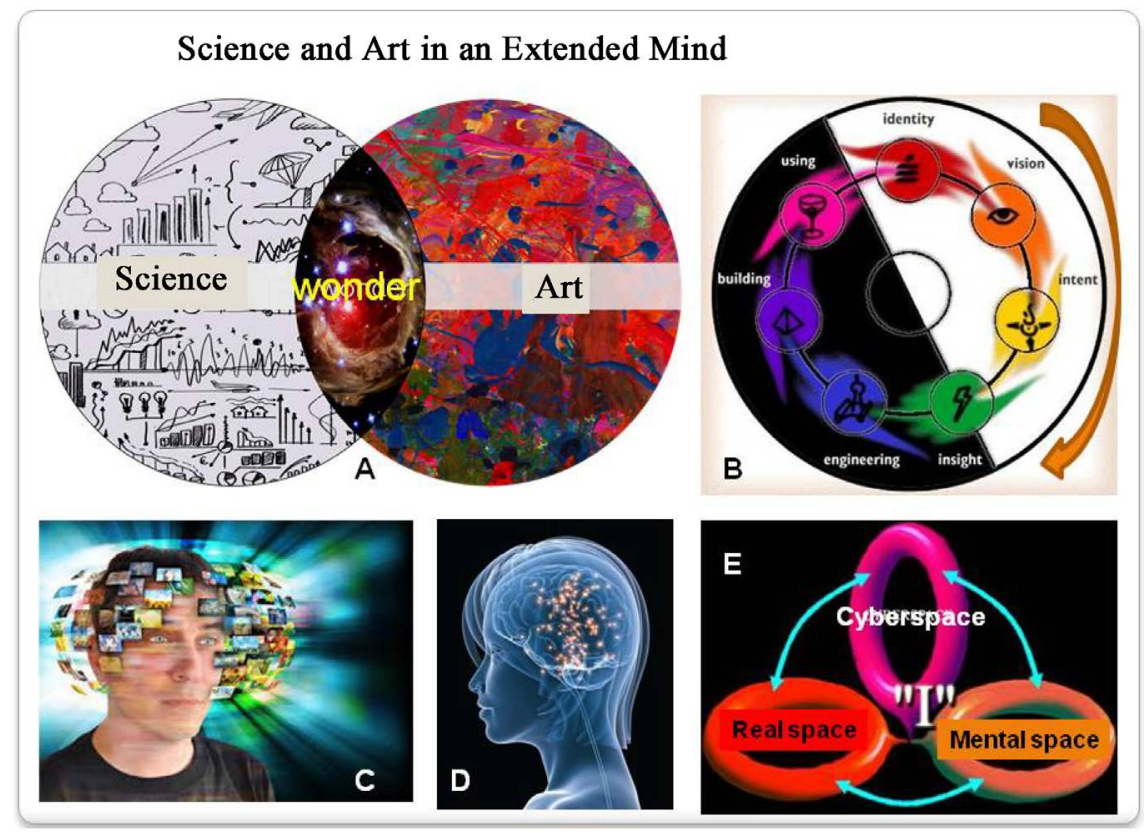

Figure 10. The superposing domains of Science and Art that create wonder (A) on the basis of the supposed cycle of creativity (B) projected in an extended modality of mind, based on multi-dimensional space models of brain function (C) and (D) in which information in mental space is interacting with that in cyberspace $(\mathrm{E})$. 
are similar to a torus. Concentrations of sufficient mass in each of the three spaces create areas that bend distance and time, like the phenomenon of a black hole. Cyberspace can thereby function as the electronic unifier of the three spaces, and metaphorically spoken, enlarges the event horizon (the boundary of the black hole) of human consciousness. Cyberspace can be envisioned as electronic space, composed of computer networks like Internet. In them information moves at high speed: less than 0.1 seconds are needed for an electronic signal to join the two most distant points on Earth. Rosen argues that thereby, the energy mass and energy of cyberspace compresses Earth to a virtual black hole, having an event horizon of approximately $2 \mathrm{~cm}$, and thus located at a near-zero distance of 0.1 seconds from us, the users connected to it. Cyberspace is thus viewed upon as the extension of the human brain that creates a new kind of earth-bound integrated consciousness. It follows that man is in an advanced stage of evolution, in a symbiotic process that unites his body with electronic and biological components. The human brain, roughly composed of three layers, (the reptilian complex, limbic system and neo-cortex) has thereby acquired another layer, the super-neo-cortex that augments the imagination and knowledge and hopefully also wisdom, and accelerates man's judgement and sense of time and space.

Evolution, in this manner, connects the human brain "event horizon" (Meijer \& Geesink, 2007) to that of cyberspace, and to real space (Shlain, 1991). Thus triangular joining of the three spaces, bends space into a closed loop, that is orbitfold-shaped (see Figure 10E). New technologies, like the neural microcircuits that link live brain neurons with electronic interface, will enable direct connection of our body with computer networks.

In fact, we humans already seem to be Cyborgs as being connected, and in the near future implanted, with electronic components. This act extends each human associative cortex to a new gigantic capacity, of the human brain. This Internet extension, or the super neo-cortex, which in turn is linked to all the other network participants, may create a united super brain with an integrated universal consciousness.

Rosen asked: "what are the implications of those insights in Cyberspace art? New media art, which utilizes digital equipment like computers is subject to physical laws. The electronic chip accelerates the artistic object, from its low speed and low mass realm outside the cyberspace event horizon, to an immediate bent, high speed and high mass cyberspace black hole medium. This transformation is typical of all technologies exploiting electromagnetic waves. The huge spread of digital cameras and cell-phones, with imbedded digital cameras on top of the PC computers, produces an audio-visual data tsunami with hundreds of billions of digital images and cell-calls per year.

The installation of parallel space by the artist therefore creates a merging of three spaces: mental, the real, and the virtual. Multiplication of art work images on computer screens all over the world reflects electro-magnetic waves that are trapped, and move in closed loops within the topology of the torus and are 
eventually emitted beyond the event horizon of cyberspace.

Kelly (2015) speculated on the future of performing science, projecting that there will be more change in the next 50 years than in the last 400 years, pointing at the way scientist will employ computer and internet in a very different manner. He mentions an array of advanced methods and procedures that will revolutionize and greatly accelerate science endeavor such as the creation and weighing of various levels of meaning from large sets of data. Other examples are compilation of so called negative results instead of throwing them away, triple blind experiments in which even investigators are blind to the goal of the experiment, combinatorial sweep exploration by analyzing millions of possible variations in for instance protein structure, as well as evolutionary search on libraries of variations in which the final result of the search is fed into a subsequent array of structure mutations. He mentions also multiple hypothesis matrices, enabling parallel testing and optimation of multiple theories, pattern augmentation in algorithmic discovery and recognition, in addition to $A I$ proofs to check the logic of experiments, as well as modalities of Wiki science with large numbers of scientists involved in a study.

\section{Conclusion}

The processes of science and art can be modeled by a recurrent spiral flux of information as represented in the topological energy trajectories of the nested torus, including knot formation. Both science and art are increasingly influenced by the rapidly rising technologies of information processing and managing. Yet, it should be realized that personal curiosity and individual creativity will always provide the driving forces for these human endeavors. Such activities require an ongoing analysis of the very nature of these versatile processes, for the sake of efficiency, quality control and long-term perspectives. For this we urgently need the restoration of our academic fundament, combined with the freedom of artistic picturing of the world we live in, to ensure a future that fulfils our hope for a better world and the survival of our splendid planet in a cosmological context.

\section{References}

Andersen, H., \& Hepburn, B. (2016). Scientific Change. Internet Encyclopedia of Philosophy. http://www.iep.utm.edu/s-change/

Banschoff, T. F. (1998). An Interactive Gallery on the Internet "Surfaces beyond the Third Dimension". International Journal of Shape Modeling.

Barr, F. (2006). The Theory of Evolutionary Process as a Unifying Paradigm. http://arthuryoung.com/barr.html

Barus, I. (2001). The Art of Science: Science of the Future in Light of Alterations in Consciousness. Journal of Scientific Exploration, 15, 57-68.

Bauer, H. W. (2014) The Science Bubble. EdgeScience, \#17, 3-6 February 2014. https://scimedskeptic.wordpress.com/2013/11/10/the-science-bubble/

Bauer, M. W., \& Suerdem, A. (2016). Relating Science Culture and Innovation. OECD Blue Sky Meeting on Science and Innovation Indicators, Ghent, September 2016. 
Bentov, I. (1979). Stalking the Wild Pendulum on the Mechanisms of Consciousness. New York: Bantam Books.

Bernstein, P. (2005). Intuition. What Science Says (So Far) about How and Why Intuition Works. In Endophysics: Time, Quantum and the Subjective. Singapore: World Science Publishing. https://doi.org/10.1142/9789812701596_0026

Bishof, M., \& Del Giudice, E. (2013). Communication and the Emergence of Collective Behaviour in Living Organisms: A Quantum Approach. Molecular Biology International, 2013, Article ID: 987549. https://doi.org/10.1155/2013/987549

Bizzari, M., Soto, A, Sonnesschein, C., \& Longo, G (2017). Saving Science. And beyond Organisms. Journal of Biological Sciences, 1.

Bjerve, A. (2016). The Fractal-Holographic Universe. http://holofractal.net/the-holofractographic

Bohm, D. F., \& Peat, D. (2008). Science, Order and Creativity (2nd ed.). Routledge (Transferred to Digital Printing Routledge).

Bohm, D., \& Hiley, B. J. (1993). The Undivided Universe. An Ontological Interpretation of Quantum Theory. London: Routledge.

Brady, R. H. (2000). Perception: Connections between Art and Science. http://www.natureinstitute.org/txt/rb/art/perception.htm

Bruza, P. D., \& Busemeyer, J. R. (2012). Quantum Models of Cognition and Decision. Cambridge: Cambridge University Press.

Butterfield, J. (2014). Our Mathematical Universe. Book Review in Plus Magazine of the UK Mathematics Millenium Project.

Davies, R. (2006). The Tyranny If Big Ideas. http://russelldavies.typepad.com/planning/2006/06/the_tyranny_of_.html

Dodig-Crnkovic, G. (2007). Chapter X: Where Do New Ideas Come From? How Do They Emerge?-Epistemology as Computation (Information Processing). Vasteras: Malardalen University. http://www.idt.mdh.se/ gdc/work/NewIdeas.pdf

Dodig-Crnkovic, G. (2012). Floridi’s Informational Structural Realist Basis for Infocomputational Modeling of Cognizing Agent, Informational Structural Realism, ISR.

Eagleman, D. M. (2013). Why Public Dissemination of Science Matters. The Journal of Neuroscience, 33, 12147-12149. https://doi.org/10.1523/JNEUROSCI.2556-13.2013

Filley, C. M. (2016). Across the Great Two-Cultures Divide. In E. R. Kandel (Ed.), Reductionisms in Art and Brain: Bridging the Two Cultures. Columbia Ininiverity Press.

Fishbein, E. (2005). Intuition in Science and Mathematics-An Educational Approach. Berlin: Springer. https://www.springer.com/la/book/9789027725066

Gaudreau, Mx. A. (2016). Unexpected Ramnification of Knot Theory. Asian Pacific Mathematics Newsletter, 6, 7-21.

http://www.asiapacific-mathnews.com/06/0601/0007_0021.pdf

Geesink, H. J., \& Meijer, D. K. F. (2018). Evidence for a Guiding Principle in Quantum Physics. Quantum Biosystems, 9, 1-7.

http://www.quantumbiosystems.org/admin/files/QBS\%209\%20(1)\%201-17\%20(2018). pdf

Geesink, J. H., \& Meijer, D. K. F. (2016a) Quantum Wave Information of Life Revealed: An Algorithm for Electromagnetic Frequencies that Create Stability of Biological Order, with Implications for Brain Function and Consciousness. NeuroQuantology, 14, 106-125. https://www.neuroquantology.com/index.php/journal/article/view/911/751

Geesink, J. H., \& Meijer, D. K. F. (2016b). Bio-Soliton Model that Predicts Non-Thermal 
Electromagnetic Frequency Bands, that Either Stabilize Living Cells. https://arxiv.org/pdf/1610.04855

Geesink, J. H., \& Meijer, D. K. F. (2017a). Bio-Soliton Model that Predicts Non-Thermal Electromagnetic Frequency Bands, that Either Stabilize Living Cells. Electromagnetic Biology and Medicine, 36, 357-378.

https://www.tandfonline.com/doi/abs/10.1080/15368378.2017.1389752?.journalCode=ie $\underline{\mathrm{bm} 20}$

Geesink, J. H., \& Meijer, D. K. F. (2017b). Electromagnetic Frequency Patterns that Are Crucial for Health and Disease Reveal a Generalized Biophysical Principle: The GM scale. Quantum Biosystems, 8, 1-16.

Gough, W. C., \& Shacklett, R. L. (2014). The Science of Connectiveness. Part 3. The Human Experience. Subtle Energies, 4, 187-214.

Grossman, L. (2012). Hawking's Escher-Verse Could Be Theory of Everything. New Scientist, 9 June 2012.

Hamlin, J. F., \& Séquin, C. H. (2009). Ribbed Surfaces for Art, Architecture and Visualization. Computer-Aided Design and Applications, 6, 1-4. https://doi.org/10.3722/cadaps.2009.749-758

Haramein, N., Brown, W. D., \& Val Baker, A. (2016). The United Spacememory Network: From Cosmogenesis to Consciousness. NeuroQuantology, 14, 1-15. https://doi.org/10.14704/nq.2016.14.4.961

Heylighen, F., \& Chielens, K. (2006). Cultural Evolution and Memetics. In Encyclopedia of Complexity and System Science. http://pespmc1.vub.ac.be/Papers/Memetics-Springer.pdf

Hofstadter, D. (2007). I Am a Strange Loop.

Holland, P. (1996) Quantum Back-Reaction and the Particle Law of Motion. http://users.ox.ac.uk/ gree0579/index_files/back-reaction.pdf

Horgan, J. (2012). What Thomas Kuhn Really Thought about Scientific Truth. Scientific American.

http://blogs.scientificamerican.com/cross-check/what-thomas-kuhn-really-thought-ab out-scientific-truth/

Huang, S. (2013). When Peers Are Not Peers and Don't Know It: The Dunning-Kruger Effect and Self-Fulfilling Prophecy in Peer-Review. Bioessays, 35, 414-416.

Institute of Venture Science. Critical Comments on Today's Scientific Enterprise. http://venturescience.wixsite.com/ivsci/todays-science

Judge, A. (2016a). Being a Poem in the Making. Engendering a Multiverse through Musing. https://www.laetusinpraesens.org/musings/muverse.php

Judge, A. (2016b). Visualization-in-3D-of-Dynamics-of-Toroidal-Helical-Coils. https://www.laetusinpraesens.org/docs10s/helixtor.php https://www.transcend.org/tms/2016/11/visualization-in-3d-of-dynamics-of-toroidal-h $\underline{\text { elical-coils/ }}$

Kastrup, B. (2017). Self-Transcendence Correlates with Brain Function Impairment. Journal of Cognition and Neuroethics, 4, 33-42.

Kauffman, L. H. (1987). State Models and the Jones Polynomial. Topology, 26, 395-307. https://doi.org/10.1016/0040-9383(87)90009-7

Kelly, K. (2015). Speculations on the Future of Science. https://www.edge.org/conversation/kevin_kelly-speculations-on-the-future-of-science

Keppler, J. A. (2013). A New Perspective on the Functioning of the Brain and the Me- 
chanisms behind Conscious Processes. Frontiers in Psychology, Theoretical and Philosophical Psychology, 1-6. https://doi.org/10.3389/fpsyg.2013.00242

Keppler, J. A. (2016). On the Universal Mechanism Underlying Conscious Systems and the Foundations for a Theory of Mind. Open Journal of Philosophy, 6, 346-367. https://doi.org/10.4236/ojpp.2016.64034

Kuhn, T. S. (1970). The Structure of Scientific Revolutions. International Encyclopedia of UNIFIED Science, 2nd Edition.

http://projektintegracija.pravo.hr/_download/repository/Kuhn_Structure_of_Scientific _Revolutions.pdf

Lambert, N., Chen, Y. N., Cheng, Y. C., Li, C. M., Chen, G. Y., \& Franco, N. (2013). Quantum Biology. Nature Physics, 9, 10-18. https://doi.org/10.1038/nphys2474

Langan, C. (2017). An Introduction to Mathematical Metaphysics. Cosmos and History. The Journal of Natural and Social Philosophy, 13, 313-330.

Laszlo, E. (2007). The Akashic Field. New York: Dutton.

Linov, (2014). Introduction to Knot Theory and the Knot Group. http://math.uchicago.edu/ may/REU2014/REUPapers/Linov.pdf

Loll, R. (2011). Quantum Gravity at the Planck Scale: Getting a Hold on Spacetime Foam. https://www.scribd.com/document/170221398/Renate-Loll

Maeda, J. (2004). Artists and Scientists: More Alike than Different. https://blogs.scientificamerican.com/guest-blog/artists-and-scientists-more-alike-thandifferent/

Malina, F. J. (1968). Some Reflections on the Differences between Science and Art. In Directions in Art Theory and Anesthetics (pp. 134-149). London: Faber.

Mathijs, E., \& Mosselmans, B. (1993). Similarity or Difference: The Case for Interdisciplinarity between Natural Sciences, Social Sciences and Art and Aesthetics. http://pespmc1.vub.ac.be/CLEA/Reports/EM-Similarities.html

Megidish, E., Halevy, A., Shacham, T., Dvir, T., Dovrat, L., \& Eisenberg, H. S. (2012). Entanglement between Photons That Have Never Coexisted. arXiv:1209.4191v1[quant-ph]

Meijer, D. K. F. (2007). Van Meme tot Medicijn. In J. van Baak (Damon) (Ed.), Over Boodschap en Beeldvorming in Cultuur en Cognitie (pp. 99-119).

Meijer, D. K. F. (2012). The Information Universe. On the Missing Link in Concepts on the Architecture of Reality. Syntropy Journal, 1, 1-64. http://www.sintropia.it/english/2012-eng-1-1.pdf

Meijer, D. K. F. (2013). Information: What Do You Mean? Syntropy Journal, 3, 1-49. http://www.lifeenergyscience.it/english/2013-eng-3-01.pdf

Meijer, D. K. F. (2014). The Extended Brain: Cyclic Information Flow, in a Quantum Physical Realm. NeuroQuantology, 12, 180-200.

http://www.neuroquantology.com/index.php/journal/article/view/754 https://doi.org/10.14704/nq.2014.12.2.754

Meijer, D. K. F. (2015). The Universe as a Cyclic Organized Information System. An Essay on the Worldview of John Wheeler. NeuroQuantology, 1, 1-40. http://www.neuroquantology.com/index.php/journal/article/view/798/693

Meijer, D. K. F., \& Geesink, J. H. (2016). Phonon Guided Biology. Architecture of Life and Conscious Perception Are Mediated by Toroidal Coupling of Phonon, Photon and Electron Information Fluxes at Discrete Eigenfrequencies. NeuroQuantology, 14, 718-755. http://www.neuroquantology.com/index.php/journal/article/view/985

Meijer, D. K. F., \& Geesink, J. H. (2017). Consciousness in the Universe Is Scale Invariant and Implies the Event Horizon of the Human Brain. NeuroQuantology, 15, 41-79. 
https://www.neuroquantology.com/index.php/journal/article/viewFile/1079/852

Merali, Z. (2017). The Universe Is a String-Net Liquid.

http://dao.mit.edu/ wen/NSart-wen.html

Merrick, R. (2008). Interference. A Grand Scientific Musical Theory. http://interferencetheory.com/Books/BooksInterference/Summary.html

Merrick, R. (2010). Harmonically Guided Evolution. Fairview.

Nickles, Th. (2014). Scientific Revolutions. In E. N. Zalta (Ed.). The Stanford Encyclopedia of Philosophy (Summer 2014 Edition).

http://plato.stanford.edu/archives/sum2014/entries/scientific-revolutions/

Niemetz, A. (2004). Singing Cells, Art, Science and Noise in between. MFA Thesis, UCLA Department of Design/Media Arts.

Perla, R. J., \& Carifo, J. (2004). The Nature of Scientific Revolutions from the Vantage Point of Chaos Theory: Toward a Formal Model of Scientific Change. Science and Education, 1-28.

Pesic, P. (2015). Music and the Making of Modern Science. http://peterpesic.com/books

Pitkänen, M. (2013). What Music Could Teach us about Consciousness. https://www.researchgate.net/publication/281198078_What_Music_Could_Teach_abo ut_Consciousness

Plsek, P. (1996). Working Paper: Models for Creative Process. http://www.directedcreativity.com/pages/WPModels.html

Popper, K. (1969). The Logic of Scientific Discovery. Harper \& Row, New York, Conjectures and Refutations. The Growth of Scientific Knowledge, RKP, London,

Primack, J., \& Abrams, N. E. (2006). The View from the Centre of the Universe. Discovering our Extraordinary Place in the Cosmos. London: Fourth Estate. http://www.chronon.org/reviews/view_from_centre.html

Purwins, H., Blankertz, B., \& Obermayer, K. (2007). Toroidal Models in Tonal Theory and Pitch-Class Analysis. In Tonal Theory for the Digital Age (Computing in Musicology 15, 2007), 73-98.

Rosen, A. (2005). Art and the Event Horizon. http://ctheory.net/ctheory_wp/art-at-the-event-horizon/

Ruse, M. (2009). The Darwinian Revolution: Rethinking Its Meaning and Significance. Proceedings of the National Academy of Sciences of the United States of America, 106, 10040-10047. https://doi.org/10.1073/pnas.0901011106

Sarewitz, D. (2016). Saving Science. Science Isn't Self-Correcting, It's Self-Destructing. To Save the Enterprise, Scientists Must Come Out of the Lab and into the Real World. The New Atlantis, 49, 4-40.

Sarfatti, A. (2015). Bohm Pilot Wave Post Quantum Theory. https://www.academia.edu/27132022/Bohm_Pilot_Wave_Post-Quantum_Theory

Scaruffi, P. (2007). Bridging the Gap between Art and Science. Notes for a Round Table Moderated by L. Shlain, San Francisco, USA.

Scientific Revolutions (2017). https://plato.stanford.edu/entries/scientific-revolutions/ https://leibniz.stanford.edu/friends/preview/scientific-revolutions/

Shlain, L. (1991). Art \& Physics: Parallel Visions in Space, Time, and Light. New York: Morrow.

Skeptical Investigations. Some Notes on Pseudo-Skepticism.

http://www.skepticalinvestigations.org/Examskeptics/skepticism_suppressedscience.html 
Smoyer, L. (2005). Musical Mathematics, the Mathematical Structure of the Pythagorean and Equal Tempered Scale. In Partial Fulfillment of the Masters of Science in Teaching Mathematics.

Sullivan, J. M. (2003). Optimal Geometry of Art. http://torus.math.uiuc.edu/jms/Essays/OptGeomArt.html

Sutherland, R. I. (2006). Causally Symmetric Bohm Model. https://pdfs.semanticscholar.org/c5f0/8198ef854f5fd824110d9aab05101879d63c.pdf

Tegmark, M. (2014). Our Mathematical Universe. My Quest for the Ultimate Nature of Reality. UK: Penquin Books Ltd.

Tozzi, A., \& Peters, J. F. (2016). Towards a Fourth Spatial Dimension of Brain Activity. Cognitive Neurodynamics, 10, 189-199. https://doi.org/10.1007/s11571-016-9379-Z

Van De Bogart, W., \& Forshaw, S. (2015). Re-Constructing Memory Using Quantized Electronic Music and a "Toridion Byte" Quantum Algorithm: Creating Images Using Zero Logic Quantum Probabilistic Neural Networks (ZLQNN). https://www.researchgate.net/publication/281480809

White, J. H. (2013). Poetry of Light. https://poetryoflight.org/2013/05/03/ouroboros/

Wilczek, F. (1989). Longing for Harmonies. Themes and Variations from Modern Physics.

https://www.amazon.com/Longing-Harmonies-Themes-Variations-Physics/dp/039330 $\underline{5961}$

Wilczek, F. (2016). A Beautiful Question, Finding Nature’s Deep Design. London: Penquin Books.

Wilkins, J. S. (1995). Evolutionary Models of Scientific Change. Master Thesis, Mount Martha, Australia.

Williamson, J. G., \& Mark van der, M. B. (1997). Is the Electron a Photon with Toroidal Topology? Annales de la Fondation Louis de Broglie, 22, 133.

Yepez, O. (2004). Matter and Light in Flatland. General Physics (physics.gen-ph). http://arxiv.org/pdf/physics/0401153.pdf

Young, A. M. (2006). Comments on Science. Essays. http://arthuryoung.com/essays.html 


\section{Annex}

It would be of great interest to finally test the present integrative toroidal Science/Art model by computer simulation, through performing experiments in which, historically recognized, contemporary scientific/artistic concepts are processed through algorithmically-based toroidal flux simulating software. Simulation of outcomes, after various cycles of iteration of such concepts, thereby inviting the recurrent interactions, could then be recorded, as have been attempted, for instance by Van de Boogart \& Forshaw, 2015 in music theory. Testability of the science model, could be approached by a processing of literature data, combined with activities and known reputations of scientific schools and, later, also individual scientists (see a relevant example in Bauer \& Suerdem, 2016) that produced relevant examples of major scientific attractors, transitions or real paradigm changes. A similar approach can be found in the current attempts to mathematically describe the phenomenon of consciousness as a process of integration of active information on the basis of an external holographic information domain with a $4 \mathrm{D}$ field receptive workspace that was proposed to be associated but not reducible to the brain itself (Meijer \& Geesink, 2017).

${ }^{\star}$ Recent work of the author see:

https://www.researchgate.net/profile/Dirk_Meijer4

\section{Further Reading}

Wikipedia/Science method

Wikipedia/Scientific revolution

Wikipedia/Models of Scientific inquiry

Wikipedia/Paradigm shift

Wikipedia/Ouroboros

Wikipedia/Implicate and explicate order

Wikipedia/Scale relativity

Wikipedia/Zero-point energy 